\title{
The motion of a deforming capsule through a corner
}

\author{
Lailai $\mathrm{Zhu}^{1,2, \dagger}$ and Luca Brandt ${ }^{1}$ \\ ${ }^{1}$ Swedish e-Science Research Centre and Linné Flow Centre, KTH Mechanics, \\ S-100 44 Stockholm, Sweden \\ ${ }^{2}$ Laboratory of Fluid Mechanics and Instabilities, Station 9, EPFL, 1105 Lausanne, Switzerland
}

(Received 22 December 2013; revised 6 February 2015; accepted 6 March 2015; first published online 8 April 2015)

\begin{abstract}
A three-dimensional deformable capsule convected through a square duct with a corner is studied via numerical simulations. We develop an accelerated boundary integral implementation adapted to general geometries and boundary conditions. A global spectral method is adopted to resolve the dynamics of the capsule membrane developing elastic tension according to the neo-Hookean constitutive law and bending moments in an inertialess flow. The simulations show that the trajectory of the capsule closely follows the underlying streamlines independently of the capillary number. The membrane deformability, on the other hand, significantly influences the relative area variations, the advection velocity and the principal tensions observed during the capsule motion. The evolution of the capsule velocity displays a loss of the time-reversal symmetry of Stokes flow due to the elasticity of the membrane. The velocity decreases while the capsule is approaching the corner, as the background flow does, reaches a minimum at the corner and displays an overshoot past the corner due to the streamwise elongation induced by the flow acceleration in the downstream branch. This velocity overshoot increases with confinement while the maxima of the major principal tension increase linearly with the inverse of the duct width. Finally, the deformation and tension of the capsule are shown to decrease in a curved corner.
\end{abstract}

Key words: biological fluid dynamics, boundary integral methods, capsule/cell dynamics

\section{Introduction}

Elastic micro-capsules are ubiquitous in nature, appearing in the form of seeds, eggs, cells and similar. The elasticity of the cells plays an important role in their proper biological functioning. As examples, red blood cells (RBC) deform significantly in micro-vessels to ease oxygen transportation; leukocytes squeeze through small gaps into the endothelial cell wall during inflammation (Springer 1994), as do tumour cells do in tumour metastasis (Hanahan \& Weinberg 2000). On the other hand, artificial micro-capsules are commonly used in the food and cosmetic industry for a controlled release of ingredients (Barthès-Biesel 2011) and synthetic nano-capsules promise a precise and targetted drug delivery. The ability of biological and artificial capsules to

$†$ Email address for correspondence: lailaizhu00@gmail.com 
dynamically adapt, change their shapes and withstand stresses from the surrounding medium has thus attracted much attention from research groups in different fields.

In micro-fluidic applications, one of the most fundamental issues is the behaviour of these tiny deformable structures when interacting with an external applied flow. Early experimental studies discovered several interesting features of RBCs: the well-known tank-treading and tumbling motion in shear flow (Goldsmith \& Marlow 1972; Fischer \& Schmid-Schönbein 1978), 'parachute' shaped deformation (Skalak \& Branemark 1969) and the 'zipper' flow pattern (Gaehtgens, Dührssen \& Albrecht 1980) in the micro-capillaries. These observations show that the capsule shape is not given a priori but determined by the dynamic balance of interfacial forces with fluid stresses. Several analytical studies deal with unbounded domains to model tank-treading and tumbling motions of an initially spherical capsule by asymptotic analysis (Barthès-Biesel 1980, 1981), prove the existence of 'slipper' shaped cells in capillary flows (Secomb \& Skalak 1982), predict the vacillating-breathing behaviour of a vesicle (Misbah 2006) and the swinging-tumbling transition of a capsule (Vlahovska et al. 2011).

Numerical simulations have been successfully used to solve the associated nonlinear fluid-structure problem; examples are the deformation of spherical (Pozrikidis 1995, 2001; Foessel et al. 2011), elliptical (Ramanujan \& Pozrikidis 1998; Walter, Salsac \& Barthès-Biesel 2011) or RBC-shaped (Pozrikidis 2003) capsules in an unbounded shear flow. However, in a realistic situation, biological cells and artificial capsules are convected in bounded channels or ducts. Motivated by early experiments showing the migration of RBCs towards the pipe centre (Goldsmith 1971), Zarda, Chien \& Skalak (1977) and Özkaya (1986) simulated the axisymmetric cellular flow in a cylindrical tube using the finite element method (FEM). Simulations based on the boundary integral method (BIM), combined with FEM for the membrane dynamics, were performed to study capsules tightly squeezed in tubes and square ducts (e.g. Hu, Salsac \& Barthès-Biesel 2011). Simulations have also addressed complex phenomena like the migration and slipper-shaped deformation of cells (Pozrikidis 2005b), suspensions of RBCs in a capillary tube (Lei et al. 2013), and the shape transition between non-axisymmetric and axisymmetric RBCs (Danker, Vlahovska \& Misbah 2009; Kaoui, Biros \& Misbah 2009). Inertial effects on the cell migration have also been investigated numerically (Doddi \& Bagchi 2008; Shi, Pan \& Glowinski 2012).

These previous computational studies focus on the capsule motion in straight geometries. However, capsules are seldom transported in such simple configurations, but rather in highly complicated capillary networks as in the in vivo micro-recirculation for RBCs or through micro-fluidic devices, where corrugations, bifurcations and corners are common. Less is known about the dynamics of capsules in these complex geometries, although these are attracting growing interest thanks to potential biomedical applications. Experiments (Braunmüller, Schmid \& Franke 2011) and simulations (Noguchi et al. 2010) have shown rich behaviours of RBCs and vesicles going through sawtooth-shaped channels; a transition from shape oscillations to orientational oscillations was identified for such deformable micro-objects, depending on the flow rate and confinement. Two-dimensional FEM computations have been carried out by Barber et al. (2008) to examine the cell partitioning in small vessel bifurcations, showing that the cells preferentially enter the branch with higher flow rate; such an effect is intensified by the cell migration towards the centre and hindered by obstructions near the bifurcations. Woolfenden \& Blyth (2011) report two-dimensional simulations of a capsule in a pressure-driven channel with a side branch. These authors found that the capsule deformation strongly 
depends on the branch angle and the cells selected different paths at the branch junction according to their deformability. Recently, Park \& Dimitrakopoulos (2013) used the spectral boundary element method to investigate the deformation of capsules and droplets passing through a sharp constriction in a square duct. These authors examine the effect of the viscosity ratio on the non-tank-treading capsule dynamics and investigate the flow circulation inside the capsule.

The flow passing around a corner is one of the most basic flow configurations; despite its universality in biological systems and micro-fluidic devices, its influence on deformable micro-objects is not fully understood. Steps in this direction have been taken only recently: the experiments by Rusconi et al. (2010) have revealed the rapid formation of bacterial streamers near the corners of a curved micro-channel at low Reynolds number due to the local vortical flow structure. This secondary flow appears as long as the curvature of the boundary varies, even in the inertialess Stokes flow (Lauga, Stroock \& Stone 2004). Simulations of an elastic filament in a two-dimensional corner flow (Autrusson et al. 2011) show that the filament crosses over the curved streamlines in the corner, instead of aligning with the flow as in a rectilinear flow. One of the motivations of this work is to assess whether the corner flow can be used to infer the material properties of soft particles as done by Lefebvre et al. (2008), Chu et al. (2011) and $\mathrm{Hu}$ et al. (2013). In those investigations, the equilibrium shape of capsules moving at a constant speed in confined channels or tubes is compared with that obtained from simulations or theory. As the corner flow is characterized by spatial non-uniformity, the capsule dynamics will undergo a transient evolution that may therefore provide additional information on the membrane properties such as viscoelasticity. Knowledge of the capsule behaviour in spatially developing flows may therefore help to explore the material properties of soft capsules.

In this work, we numerically study the motion and deformation of an individual capsule transported in a duct with a straight and/or a curved corner. A threedimensional code is developed to compute the motion of deformable capsules in arbitrary configurations. This is based on a boundary integral formulation with Ewald acceleration as suggested by Hernández-Ortiz, de Pablo \& Graham (2007); the method shares the elegance of both boundary integral and mesh-based methods. Boundary integrals are computed to accurately account for the singular and fast-varying interactions while the smooth part of the solution is handled by a highly parallel general Stokes solver based on the spectral element method. The integration on the membrane is based on a global spectral surface interpolation using spherical harmonics (Zhao et al. 2010). Our hybrid scheme couples, therefore, the high accuracy of boundary integrals for the short-ranged interactions to the geometrical flexibility of mesh-based methods (Freund 2014). Spherical harmonics are utilized to resolve the membrane dynamics with spectral accuracy. The same implementation has been used to simulate cell sorting by deformability in a micro-fluidic device of complex geometry, i.e. a semi-cylindrical pillar embedded in a divergent channel (Zhu et al. 2014).

The paper is organized as follows. The geometrical setup and the numerical method are described in $\S 2$. The results are presented in $\S 3$ and their discussion and a summary of the main conclusions is provided in $\S 4$.

\section{Problem setup and numerical method}

\subsection{Flow geometry and numerical procedure}

Figure 1 displays the flow configuration and the coordinate system used in the present investigation, where half of the flow domain is removed to better visualize 
(a)

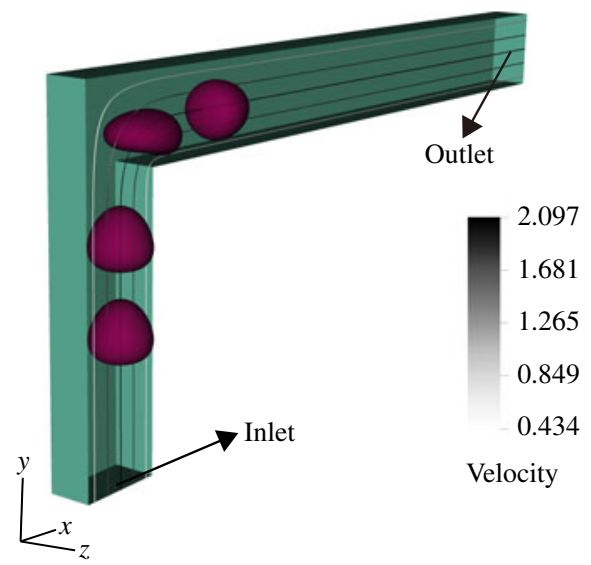

(b)

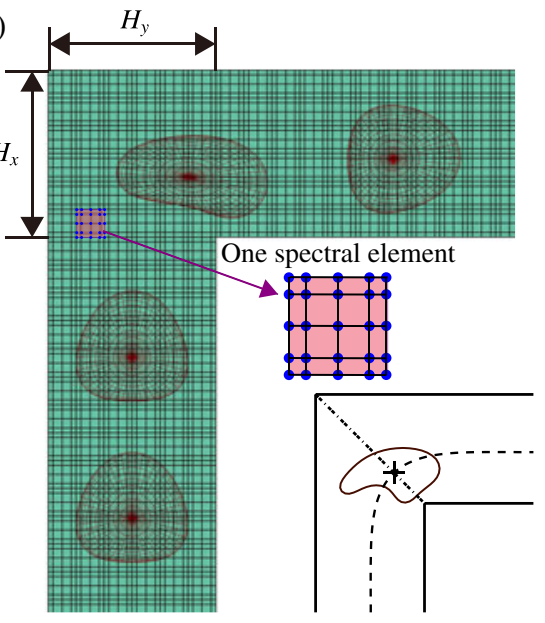

FIgURE 1. (Colour online) Flow configuration. (a) A deformable capsule travelling in a square duct with a $90^{\circ}$ straight corner, with half of the domain displayed. The analytical velocity profile is imposed at the inlet with a maximum centreline velocity of 2.097. The flow field without capsules is depicted by the streaklines and the grey-scale colour indicates the velocity magnitude. (b) The discretized fluid domain and the capsule at four positions on the $x-y$ mid-plane. The box with dots represents one spectral element with $5 \times 5 \times 5$ GLL points. In the inset at bottom right, the dashed line denotes the trajectory of the capsule, the dot-dashed line the axis of geometrical symmetry and the cross the capsule centre when located on the axis at instant $t=0$.

the deforming capsule. We investigate the motion of an elastic capsule transported through a square duct of width $H=H_{x}=H_{y}$; we keep $H_{x}=H_{y}$ in this work. In the figure, the streaklines and grey-scale contours, coded by the velocity magnitude, are shown on the $x-y(z=0$, omitted hereinafter $)$ mid-plane. The duct is characterized by a $90^{\circ}$ straight corner.

We consider an initially spherical capsule of radius $a$, enclosed by an infinitely thin hyperelastic membrane with surface shear modulus $G_{s}$. The fluid inside and outside the capsule has the same density $\rho_{F}$ and viscosity $\mu$; buoyancy forces and sedimentation effects are neglected.

As capsules are usually small, the Reynolds number $R e$ defined with the capsule radius $a$ and the characteristic flow velocity $V_{C}, R e=\rho_{F} V_{C} a / \mu \ll 1$. Viscous forces are therefore dominant over inertial forces, and the flow inside and outside the capsule is governed by the linear Stokes equations and determined instantaneously by the boundary conditions. A proper tool to solve the problem is therefore the BIM and we adopt here an accelerated variant of it.

The fluid-structure interaction problem is solved as follows: the flow convects and distorts the capsule while the restoring elastic forces alter the fluid motion (Walter et al. 2010). We start with an undeformed capsule near the inflow and compute, at each time step, the elastic force on the membrane from the deformed (and out-ofequilibrium) shape of the capsule. Neglecting inertia and Brownian fluctuations, the force density exerted by the capsule onto the fluid is equal to the membrane load. Given this forcing, the velocities of the membrane nodes are computed explicitly with the BIM (see the following section). 


\subsection{Numerical method}

\subsubsection{Accelerated BIM}

We develop a boundary integral implementation accelerated by the general-geometry Ewald-like method (GGEM), proposed by Hernández-Ortiz et al. (2007) and later used in a variety of micro-multiphase simulations (Pranay et al. 2010; Kumar \& Graham 2011). An introduction is given here; readers are referred to the above-mentioned articles for more details. The surface of the capsule $S$ is discretized by $M$ points, the Lagrangian mesh points. The elastic force per unit area on the membrane out of equilibrium is denoted $f^{e}$. The force per unit area from the fluid to the membrane is $\boldsymbol{f}^{f}$, with $\boldsymbol{f}^{f}+\boldsymbol{f}^{e}=0$ due to the stress continuity. In return, the force per unit volume exerted by the deforming surface onto the fluid at position $\boldsymbol{x}$ is $\boldsymbol{\rho}(\boldsymbol{x})=\int_{S}-\boldsymbol{f}^{f} \delta\left(\boldsymbol{x}-\boldsymbol{x}_{m}\right) \mathrm{d} S\left(\boldsymbol{x}_{m}\right)=\int_{S} \boldsymbol{f}^{e} \delta\left(\boldsymbol{x}-\boldsymbol{x}_{m}\right) \mathrm{d} S\left(\boldsymbol{x}_{m}\right)$, with $\delta$ the Dirac delta function and $\boldsymbol{x}_{m}$ the integration variable, indicating that the integration is performed on the membrane surface. We thus need to solve the following equations for the fluid in the inertialess Stokes regime:

$$
\begin{gathered}
-\nabla p(\boldsymbol{x})+\mu \nabla^{2} \boldsymbol{u}(\boldsymbol{x})+\int_{S} \boldsymbol{f}^{e}\left(\boldsymbol{x}_{m}\right) \delta\left(\boldsymbol{x}-\boldsymbol{x}_{m}\right) \mathrm{d} S\left(\boldsymbol{x}_{m}\right)=0, \\
\nabla \cdot \boldsymbol{u}(\boldsymbol{x})=0,
\end{gathered}
$$

where $p$ and $\boldsymbol{u}$ denote the pressure and fluid velocity and $\mu$ is the fluid dynamic viscosity. Owing to the linearity of the Stokes problem, the flow field can be expressed as a boundary integral on the surface of the capsule only,

$$
\boldsymbol{u}(\boldsymbol{x})=\boldsymbol{u}^{\infty}(\boldsymbol{x})+\int_{S} \boldsymbol{G}\left(\boldsymbol{x}, \boldsymbol{x}_{m}\right) \cdot \boldsymbol{f}^{e}\left(\boldsymbol{x}_{m}\right) \mathrm{d} S\left(\boldsymbol{x}_{m}\right),
$$

where $\boldsymbol{u}^{\infty}(\boldsymbol{x})$ is the velocity field of the undisturbed flow and $\boldsymbol{G}\left(\boldsymbol{x}^{\prime}, \boldsymbol{y}^{\prime}\right)$ is the free-space Green's function of the Stokes problem, also known as the Stokeslet or Oseen-Burgers tensor,

$$
\boldsymbol{G}\left(\boldsymbol{x}^{\prime}, \boldsymbol{y}^{\prime}\right)=\frac{1}{8 \pi \mu r}\left(\boldsymbol{\delta}+\frac{\left(\boldsymbol{x}^{\prime}-\boldsymbol{y}^{\prime}\right)\left(\boldsymbol{x}^{\prime}-\boldsymbol{y}^{\prime}\right)}{r^{2}}\right),
$$

with $r=\left|\boldsymbol{x}^{\prime}-\boldsymbol{y}^{\prime}\right|$.

The GGEM method decomposes the force per unit volume $\rho(x)$ into a local part $\boldsymbol{\rho}^{l}(\boldsymbol{x})$ and a global part $\boldsymbol{\rho}^{g}(\boldsymbol{x})$, with $\boldsymbol{\rho}(\boldsymbol{x})=\boldsymbol{\rho}^{l}(\boldsymbol{x})+\boldsymbol{\rho}^{g}(\boldsymbol{x})$ and

$$
\begin{gathered}
\boldsymbol{\rho}^{l}(\boldsymbol{x})=\int_{S} \boldsymbol{f}^{e}\left(\boldsymbol{x}_{m}\right)\left[\delta-g\left(\boldsymbol{x}-\boldsymbol{x}_{m}\right)\right] \mathrm{d} S\left(\boldsymbol{x}_{m}\right), \\
\boldsymbol{\rho}^{g}(\boldsymbol{x})=\int_{S} \boldsymbol{f}^{e}\left(\boldsymbol{x}_{m}\right) g\left(\boldsymbol{x}-\boldsymbol{x}_{m}\right) \mathrm{d} S\left(\boldsymbol{x}_{m}\right),
\end{gathered}
$$

where $g\left(\boldsymbol{x}^{\prime}\right)$ is a quasi-Gaussian function used to smooth the Dirac delta function,

$$
g\left(\boldsymbol{x}^{\prime}\right)=\left(\alpha_{\text {cut }}^{3} / \pi^{3 / 2}\right) \exp \left(-\alpha_{\text {cut }}^{2}\left|\boldsymbol{x}^{\prime}\right|^{2}\right)\left[5 / 2-\alpha_{\text {cut }}^{2}\left|\boldsymbol{x}^{\prime}\right|^{2}\right],
$$

where $\alpha_{c u t}^{-1}$ indicates the length scale over which the smoothing is active.

The Stokes problem in (2.1) is therefore decomposed into two problems: one for the flow induced by the local force $\boldsymbol{\rho}^{l}(\boldsymbol{x})$, hence called the local problem, and one for its global counterpart $\boldsymbol{\rho}^{g}(\boldsymbol{x})$. The velocity field $\boldsymbol{u}(\boldsymbol{x})$ is the sum of the local velocity 
$\boldsymbol{u}^{l}(\boldsymbol{x})$ and global velocity $\boldsymbol{u}^{g}(\boldsymbol{x}), \boldsymbol{u}(\boldsymbol{x})=\boldsymbol{u}^{l}(\boldsymbol{x})+\boldsymbol{u}^{g}(\boldsymbol{x})$. The local problem accounts for the singular and short-ranged interaction while the global problem accounts for the smooth and long-ranged interactions. These are solved by different numerical methods; the local solution is calculated by the BIM due to its superior accuracy in resolving fast-decaying interactions, while the global problem is handled by a mesh-based solver that provides geometrical flexibility.

The modified Stokeslet pertaining the local problem can be shown to be

$$
\boldsymbol{G}^{\prime}\left(\boldsymbol{x}^{\prime}\right)=\frac{1}{8 \pi \mu}\left(\boldsymbol{\delta}+\frac{\boldsymbol{x}^{\prime} \boldsymbol{x}^{\prime}}{\left|\boldsymbol{x}^{\prime}\right|^{2}}\right) \frac{\operatorname{erfc}\left(\alpha_{\text {cut }}\left|\boldsymbol{x}^{\prime}\right|\right)}{\left|\boldsymbol{x}^{\prime}\right|}-\frac{1}{8 \pi \mu}\left(\boldsymbol{\delta}-\frac{\boldsymbol{x}^{\prime} \boldsymbol{x}^{\prime}}{\left|\boldsymbol{x}^{\prime}\right|^{2}}\right) \frac{2 \alpha_{\text {cut }}}{\pi^{1 / 2}} \exp \left(-\alpha_{\text {cut }}^{2}\left|\boldsymbol{x}^{\prime}\right|^{2}\right),
$$

so that the velocity field $\boldsymbol{u}^{l}(\boldsymbol{x})$ of the local solution can be obtained as

$$
\boldsymbol{u}^{l}(\boldsymbol{x})=\int_{S} \boldsymbol{G}^{l}\left(\boldsymbol{x}, \boldsymbol{x}_{m}\right) \cdot \boldsymbol{f}^{e}\left(\boldsymbol{x}_{m}\right) \mathrm{d} S\left(\boldsymbol{x}_{m}\right) .
$$

Equation (2.9) can be integrated by classical boundary integral implementations. Regularized Stokeslets can be used to facilitate the calculations, as done in, among others, Hernández-Ortiz et al. (2007) and Pranay et al. (2010). Nonetheless, the BIM with regularization suffers a degradation of the numerical accuracy and robustness for cases involving strong confinement or closely packed objects. Singular and nearly singular integration is necessary to achieve the required accuracy in these cases (Huang \& Cruse 1993; Zhu, Lauga \& Brandt 2013), and this is the approach pursued here.

As the modified Stokeslet $\boldsymbol{G}^{l}\left(\boldsymbol{x}^{\prime}\right)$ decays rapidly, the integral of (2.9) is assumed to be zero when the distance between $\boldsymbol{x}$ and $\boldsymbol{x}_{m}$ is above a cut-off radius $R_{\text {cut }}$. We choose $R_{\text {cut }}=4 \alpha_{\text {cut }}^{-1}$ and $\alpha_{\text {cut }}=a^{-1}$ as in the work of Pranay et al. (2010). The alternative value $R_{\text {cut }}=5 \alpha_{\text {cut }}^{-1}$ has also been tested for some of the cases and no significant differences have been observed.

The modified Stokeslet $\boldsymbol{G}^{l}\left(\boldsymbol{x}^{\prime}\right)$ is valid for an unbounded domain, thus the local velocity $\boldsymbol{u}^{l}(\boldsymbol{x})$ does not account for the influence of any additional boundaries. The global velocity will therefore be defined in such a way that the sum of the two will satisfy the required boundary conditions: no slip at the solid wall $\Omega$ in the cases investigated here, $\boldsymbol{u}^{l}\left(\boldsymbol{x}_{\Omega}\right)+\boldsymbol{u}^{g}\left(\boldsymbol{x}_{\Omega}\right)=0$. The global problem amounts to solving the Stokes problem in the domain of interest with the known volume forcing $\boldsymbol{\rho}^{g}(\boldsymbol{x})$ and boundary conditions defined by the solution of the local problem. This allows the use of a variety of efficient and accurate numerical methods for the solution of the Stokes equations in any complex geometry. Here, we compute the global solution with Stokes module of the open-source Navier-Stokes solver NEK5000 (Fischer, Lottes \& Kerkemeier 2008b), using the spectral element method. NEK5000 has been extensively used for stability analysis (Schrader et al. 2010) and turbulent flows (Fischer et al. 2008a) in complex domains. Akin to the FEM, the physical domain is decomposed into elements, with each element subdivided into arrays of Gauss-Lobatto-Legendre (GLL) nodes for the velocity and Gauss-Legendre (GL) nodes for the pressure field. The Galerkin approximation is employed for the spatial discretization with different velocity and pressure spaces, the so-called $\mathbb{P}_{N}-\mathbb{P}_{N-2}$ approach (Maday \& Patera 1989). Accordingly, the velocity (respectively pressure) space consists of $N$ th(respectively $(N-2)$ th-) order Lagrange polynomial interpolants, defined on the GLL (respectively GL) quadrature points in each element. Note that we do not solve the Navier-Stokes equations with a very small but finite Reynolds number, but instead 
use the steady Stokes solver of NEK5000 at each time step. NEK5000 is chosen here for its spectral accuracy, high parallel performance and most importantly its geometric flexibility, fully exploiting the general-geometry merit of GGEM.

The global problem is solved only on the Eulerian mesh points, which do not necessarily coincide with the Lagrangian mesh points on the membrane (see figure 1). Thus, at each time step, an interpolation from the global solution is performed to obtain the global velocity $\boldsymbol{u}^{g}\left(\boldsymbol{x}_{i}\right), \quad i=1,2,3, \ldots, M$, of the Lagrangian points. The interpolation error is minimized thanks to the spectral accuracy of NEK5000. The velocities of the Lagrangian points are obtained by summing up the local and global velocities. We use a third-order Adam-Bashforth time-integration scheme to update the position of those points.

\subsubsection{Spectral method for the membrane dynamics}

The membrane loading was calculated as linear piece-wise functions on triangular meshes by Pozrikidis (1995), Ramanujan \& Pozrikidis (1998) and Li \& Sarkar (2008) among others. FEM has also been implemented by Walter et al. (2010) for its generality and versatility. Bi-cubic B-splines interpolation functions were adopted by Lac, Morel \& Barthès-Biesel (2007) to obtain accurate results at a reasonably high computational cost. Alternatively, an accurate spectral boundary element algorithm was used by Dodson \& Dimitrakopoulos (2009), Kuriakose \& Dimitrakopoulos (2011, 2013), thus coupling the numerical accuracy of the spectral method and the geometric flexibility of the boundary element method. Another attractive alternative is the global spectral method. Fourier spectral interpolation and spherical harmonics have been used for two-dimensional (Freund 2007) and three-dimensional simulations (Kessler, Finken \& Seifert 2008; Zhao et al. 2010). Here, we follow the approach of Zhao et al. (2010), briefly outlined below.

We map the capsule surface onto the surface of the unit reference sphere $\mathbb{S}^{2}$, using its spherical angles $(\theta, \phi)$ for the parametrization. The parameter space $\{(\theta, \phi) \mid 0 \leqslant$ $\theta \leqslant \pi, 0 \leqslant \phi \leqslant 2 \pi\}$ is discretized by a quadrilateral grid consisting of GL quadrature points in $\theta$ and uniform intervals in $\phi$. All other surface quantities are defined on the same mesh. The surface coordinates $\boldsymbol{x}(\theta, \phi)$ are expressed by a truncated series of spherical harmonic functions,

$$
\boldsymbol{x}(\theta, \phi)=\sum_{n=0}^{N_{S H}} \sum_{m=0}^{n} \bar{P}_{n}^{m}(\cos \theta)\left(\boldsymbol{a}_{n m} \cos m \phi+\boldsymbol{b}_{n m} \sin m \phi\right),
$$

yielding $N_{S H}^{2}$ spherical harmonic modes. The corresponding normalized Legendre polynomials are

$$
\bar{P}_{n}^{m}(x)=\frac{1}{2^{n} n !} \sqrt{\frac{(2 n+1)(n-m) !}{2(n+m) !}}\left(1-x^{2}\right)^{m / 2} \frac{\mathrm{d}^{n+m}}{\mathrm{~d} x^{n+m}}\left(x^{2}-1\right)^{n} .
$$

Both forward and backward transformations are calculated with the SPHEREPACK library (Adams \& Swarztrauber 1997; Swarztrauber \& Spotz 2000). Aliasing errors arise due to the nonlinearities induced by the membrane model and the complicated geometry (products, roots and inverse operations needed to calculate the geometric quantities introduced below). We implement an approximate dealiasing by performing the nonlinear operations on $M_{S H}>N_{S H}$ points and filtering the result back to $N_{S H}$ points. A detailed discussion on this issue is provided in Freund \& Zhao (2010). 
A point on the surface is expressed by the curvilinear coordinates, $\left(\xi^{1}, \xi^{2}\right)=(\theta, \phi)$, defined on the covariant base, $\left(\boldsymbol{a}_{1}, \boldsymbol{a}_{2}, \boldsymbol{a}_{3}\right)$, following the local deformation. The base vectors are

$$
\boldsymbol{a}_{1}=\frac{\partial \boldsymbol{x}}{\partial \theta}, \quad \boldsymbol{a}_{2}=\frac{\partial \boldsymbol{x}}{\partial \phi}, \quad \boldsymbol{a}_{3}=\boldsymbol{n}=\frac{\boldsymbol{a}_{1} \times \boldsymbol{a}_{2}}{\left|\boldsymbol{a}_{1} \times \boldsymbol{a}_{2}\right|},
$$

and the covariant and contravariant metric tensors

$$
a_{\alpha \beta}=\boldsymbol{a}_{\alpha} \cdot \boldsymbol{a}_{\beta}, \quad a^{\alpha \beta}=\boldsymbol{a}^{\alpha} \cdot \boldsymbol{a}^{\beta},
$$

where $\alpha, \beta=1,2$. The base vectors and metric tensors are also defined for the undeformed state and denoted here by capital letters $\left(A^{\alpha}, A^{\alpha \beta}\right)$.

The second fundamental form coefficient of the surface is $b_{\alpha \beta}=\boldsymbol{n} \cdot\left(\partial \boldsymbol{a}_{\alpha} / \partial \xi^{\beta}\right)$ and the two invariants of the transformation $I_{1}$ and $I_{2}$ are defined as

$$
I_{1}=A^{\alpha \beta} a_{\alpha \beta}-2, \quad I_{2}=\left|A^{\alpha \beta}\right|\left|a_{\alpha \beta}\right|-1 .
$$

$I_{1}$ and $I_{2}$ can also be determined from the principal dilations $\lambda_{1}$ and $\lambda_{2}$,

$$
I_{1}=\lambda_{1}^{2}+\lambda_{2}^{2}-2, \quad I_{2}=\lambda_{1}^{2} \lambda_{2}^{2}-1=J_{2}^{2}-1 .
$$

The Jacobian, $J_{s}=\lambda_{1} \lambda_{2}$, shows the ratio of the deformed to the undeformed surface area. We compute the in-plane Cauchy stress tensor $\boldsymbol{T}$, from the strain energy function per unit area of the undeformed membrane, $W_{S}\left(I_{1}, I_{2}\right)$,

$$
\boldsymbol{T}=\frac{1}{J_{s}} \boldsymbol{F} \cdot \frac{\partial W_{S}}{\partial \boldsymbol{e}} \cdot \boldsymbol{F}^{\mathrm{T}},
$$

where $\boldsymbol{F}$ is $\boldsymbol{a}_{\alpha} \otimes \boldsymbol{A}^{\alpha}$ and $\boldsymbol{e}=\left(\boldsymbol{F}^{\mathrm{T}} \cdot \boldsymbol{F}-\boldsymbol{I}\right) / 2$ is the Green-Lagrange stain tensor. Equation (2.16) can be further expressed by components as

$$
T^{\alpha \beta}=\frac{2}{J_{s}} \frac{\partial W_{S}}{\partial I_{1}} A^{\alpha \beta}+2 J_{s} \frac{\partial W_{S}}{\partial I_{2}} a^{\alpha \beta} .
$$

We employ a widely used model of the strain energy function $W_{S}$ in our study, the neo-Hookean law (NH) (Green \& Adkins 1970) formulated as

$$
W_{S}^{N H}=\frac{G_{s}}{2}\left(I_{1}-1+\frac{1}{I_{2}+1}\right),
$$

where $G_{s}$ is the surface shear modulus. The local equilibrium connects $\boldsymbol{T}$ with the external membrane load $\boldsymbol{q}$, as

$$
\nabla_{s} \cdot \boldsymbol{T}+\boldsymbol{q}=0
$$

where $\left(\nabla_{s} \cdot\right)$ is the surface divergence operator in the deformed state. In curvilinear coordinates, the load vector is written as $\boldsymbol{q}=q^{\beta} \boldsymbol{a}_{\beta}+q^{n} \boldsymbol{n}, \beta=1,2$. The local balance in (2.19) is further decomposed into tangential and normal components,

$$
\begin{gathered}
\frac{\partial T^{\alpha \beta}}{\partial \xi^{\alpha}}+\Gamma_{\alpha \lambda}^{\alpha} T^{\lambda \beta}+\Gamma_{\alpha \lambda}^{\beta} T^{\alpha \lambda}+q^{\beta}=0, \quad \beta=1,2, \\
T^{\alpha \beta} b_{\alpha \beta}+q^{n}=0,
\end{gathered}
$$

where $\Gamma_{\alpha \lambda}^{\beta}$ are the Christoffel symbols. 
We incorporate bending stiffness into our model using the linear isotropic model for the bending moment $\boldsymbol{M}: M_{\beta}^{\alpha}=-G_{B}\left(b_{\beta}^{\alpha}-B_{\beta}^{\alpha}\right)$, where $G_{B}$ is the bending modulus, and $b_{\beta}^{\alpha}$ is the mixed version of the second fundamental form coefficients ( $B_{\beta}^{\alpha}$ corresponds to that of the reference configuration). Considering the local torque balance with bending moments exerted on the membrane, we obtain the transverse shear vector $\boldsymbol{Q}$ and in-plane stress tensor $\boldsymbol{T}$,

$$
\begin{gathered}
M_{\mid \alpha}^{\alpha \beta}-Q^{\beta}=0, \\
\boldsymbol{\varepsilon}_{\alpha \beta}\left(T^{\alpha \beta}-b_{\gamma}^{\alpha} M^{\gamma \beta}\right)=0,
\end{gathered}
$$

where ' $\mid \alpha$ ' denotes the covariant derivative and $\boldsymbol{\varepsilon}$ the two-dimensional Levi-Civita tensor. Equation (2.23) determines the antisymmetric part of the in-plane stress tensor, which is always zero as proved in Zhao et al. (2010). Including the transverse shear stress $\boldsymbol{Q}$, the local equilibrium of the stress, including bending, gives

$$
\begin{gathered}
\frac{\partial T^{\alpha \beta}}{\partial \xi^{\alpha}}+\Gamma_{\alpha \lambda}^{\alpha} T^{\lambda \beta}+\Gamma_{\alpha \lambda}^{\beta} T^{\alpha \lambda}-b_{\alpha}^{\beta} Q^{\alpha}+q^{\beta}=0, \quad \beta=1,2, \\
T^{\alpha \beta} b_{\alpha \beta}+Q_{\mid \alpha}^{\alpha}+q^{n}=0 .
\end{gathered}
$$

\subsubsection{Singular and nearly singular integration}

In this section, we report the scheme for singular and nearly singular integration based on the spectral surface discretization. We mostly follow the approach in Zhao et al. (2010), which is briefly described here for the sake of completeness. We rewrite the boundary integral equation (2.9) in its general form as

$$
\mathscr{I}\left(\boldsymbol{x}_{0}\right)=\int_{S} K\left(\boldsymbol{x}, \boldsymbol{x}_{0}\right) g(\boldsymbol{x}) \mathrm{d} S(\boldsymbol{x})=\int_{\mathbb{S}^{2}} K\left(\boldsymbol{x}(\theta, \phi), \boldsymbol{x}_{0}\right) g(\boldsymbol{x}(\theta, \phi)) J(\theta, \phi) \mathrm{d} \theta \mathrm{d} \phi,
$$

where $K$ is one component of the Green's function kernel, the modified Stokeslet in (2.8) in our case, $g$ is a smooth function defined in $S$ and $J=|(\partial \boldsymbol{x} / \partial \theta) \times(\partial \boldsymbol{x} / \partial \phi)|$ the Jacobian. If the point $\boldsymbol{x}_{0}$ is sufficiently far from the membrane surface $S, K$ is smooth and the integral $\mathscr{I}\left(\boldsymbol{x}_{0}\right)$ can be computed as

$$
\mathscr{I}\left(\boldsymbol{x}_{0}\right)=\sum_{k=1}^{M=N_{S H} \times 2 N_{S H}} K\left(\boldsymbol{x}_{k}, \boldsymbol{x}_{0}\right) g\left(\boldsymbol{x}_{k}\right) J\left(\theta_{k}, \phi_{k}\right) \omega_{k},
$$

where $\omega_{k}$ is the weight of the $k$ th discretized point. If $\boldsymbol{x}_{0}$ lies on the boundary $S$, the kernel function $K\left(\boldsymbol{x}, \boldsymbol{x}_{0}\right)$ becomes singular: in this case, a naive integration using (2.27) would give low accuracy; this so-called singular integration needs a special treatment. As $\boldsymbol{x}_{0}$ is very close to $S, K\left(\boldsymbol{x}, \boldsymbol{x}_{0}\right)$ becomes nearly singular, also requiring additional care. We adopt here the approach denoted as floating partition of unity (Bruno \& Kunyansky 2001). In the singular case, $\boldsymbol{x}_{0}$ on the surface, we define $s\left(\boldsymbol{x}, \boldsymbol{x}_{0}\right)$ as the contour length along the great circle connecting $\boldsymbol{x}$ and $\boldsymbol{x}_{0}$ on the reference sphere $\mathbb{S}^{2}$. This is used to define a mask function $\eta\left(s\left(\boldsymbol{x}, \boldsymbol{x}_{0}\right)\right)$,

$$
\eta(s)= \begin{cases}\exp \left(\frac{2 \exp (-1 / t)}{t-1}\right) & \text { if } t=s / s_{\text {cut }}<1, \\ 0 & \text { if } s \geqslant s_{\text {cut }},\end{cases}
$$


where $s_{\text {cut }}$ is a cut-off radius. With the mask function $\eta(s)$, the boundary integral $\mathscr{I}\left(\boldsymbol{x}_{0}\right)$ is decomposed into two parts, a singular part $\mathscr{I}_{\text {singular }}\left(\boldsymbol{x}_{0}\right)$ and a smooth part $\mathscr{I}_{\text {smooth }}\left(\boldsymbol{x}_{0}\right)$ :

$$
\begin{gathered}
\mathscr{I}\left(\boldsymbol{x}_{0}\right)=\mathscr{I}_{\text {singular }}\left(\boldsymbol{x}_{0}\right)+\mathscr{I}_{\text {smooth }}\left(\boldsymbol{x}_{0}\right), \\
\mathscr{I}_{\text {singular }}\left(\boldsymbol{x}_{0}\right)=\int_{S} \eta\left(s\left(\boldsymbol{x}, \boldsymbol{x}_{0}\right)\right) K\left(\boldsymbol{x}, \boldsymbol{x}_{0}\right) g(\boldsymbol{x}) \mathrm{d} S(\boldsymbol{x}), \\
\mathscr{I}_{\text {smooth }}\left(\boldsymbol{x}_{0}\right)=\int_{S}\left[1-\eta\left(s\left(\boldsymbol{x}, \boldsymbol{x}_{0}\right)\right)\right] K\left(\boldsymbol{x}, \boldsymbol{x}_{0}\right) g(\boldsymbol{x}) \mathrm{d} S(\boldsymbol{x}) .
\end{gathered}
$$

The integrand of the smooth part becomes zero when $\boldsymbol{x}$ and $\boldsymbol{x}_{0}$ coincide so that the integral can be computed accurately using (2.27). The singular part has non-zero values only on the spherical patch of radius $s_{c u t}$, and it can be integrated using local polar coordinates defined on that patch,

$$
\mathscr{I}_{\text {singular }}\left(\boldsymbol{x}_{0}\right)=\int_{0}^{2 \pi} \int_{0}^{s_{c u t}} \eta(s) K\left(\boldsymbol{x}(s, \psi), \boldsymbol{x}_{0}\right) g(s, \psi) \boldsymbol{J}^{\prime}(s, \psi) \mathrm{d} s \mathrm{~d} \psi,
$$

where $J^{\prime}(s, \psi)=|(\partial \boldsymbol{x} / \partial s) \times(\partial \boldsymbol{x} / \partial \psi)|$ is the Jacobian of the transformation. We apply Gauss quadrature along the radial direction $s \in\left[0, s_{\text {cut }}\right]$ and sum over the circumferential direction $\psi \in[0,2 \pi]$. The radius of the patch is chosen to be $s_{c u t}=\pi / \sqrt{N_{S H}}$ (see the detailed discussion in Zhao et al. 2010). Because the quadrature points do not necessarily coincide with the discretization points, interpolation is needed to obtain quantities such as $g(s, \psi)$. Bi-cubic spline interpolation is performed here: firstly, we compute $g$ on a uniform mesh in $\theta$ and $\phi$ based on the spherical harmonic coefficients; the mesh is then extended from $\theta \in[0, \pi]$ to $\theta \in[0,2 \pi]$ exploiting the symmetry $g(2 \pi-\theta, \pi+\phi)=g(\theta, \phi) ; g$ is periodic in both directions on the extended domain and its derivatives can be accurately computed by Fourier transform; we finally construct the bi-cubic spline approximation using the function derivatives.

For the nearly singular integration, we first find the projection of $x_{0}$ onto the membrane surface, $\boldsymbol{x}_{0}^{\text {proj }}$, and then compute the boundary integral on the spherical patch centred at $x_{0}^{p r o j}$. A sinh transformation is applied in the radial direction in order to move the quadrature points closer to $\boldsymbol{x}_{0}^{\text {proj }}$ (Johnston \& Elliott 2005), better resolving the fast-varying Green's function near $\boldsymbol{x}_{0}^{\text {proj }}$.

\subsection{Non-dimensionalization}

The capsule membrane is characterized by its resistance to shearing and bending. The capillary number $\mathrm{Ca}$, the ratio of viscous over elastic forces, is defined based on the surface shear modulus $G_{s}$,

$$
C a=\frac{\mu V_{C}}{G_{s}},
$$

where we use the mean velocity as the characteristic flow velocity $V_{C}$. The reduced bending modulus, $C b$, is the ratio of the bending and shearing moduli, $C b=G_{B} / a^{2} G_{s}$. We use the radius of the capsule $a$ as the reference length scale, so that the characteristic time scale is $T=a / V_{C}$. 

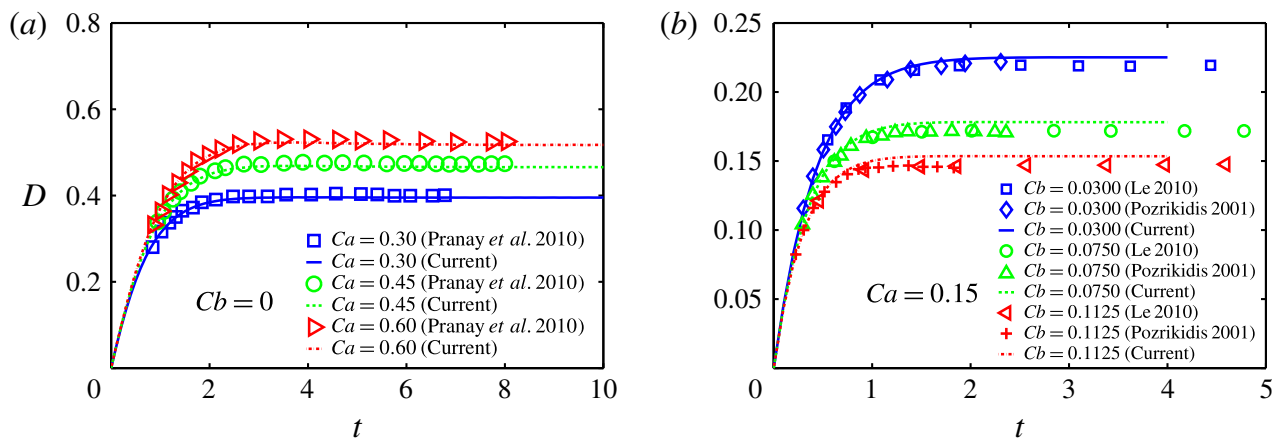

FIGURE 2. (Colour online) (a) Variation of the deformation parameter $D$ versus time for an initially spherical neo-Hookean capsule in shear flow. Different values of the capillary number $C a$ are chosen. The profile of the capsule in the shear plane is an ellipse with a long axis $L_{\max }$ and short axis $L_{\min }$; the Taylor parameter quantifying the capsule deformation is $D=\left(L_{\max }-L_{\min }\right) /\left(L_{\max }+L_{\min }\right)$. (b) Same as $(a)$, but with $C a=0.15$ and different values of the reduced bending modulus $C b$.

\subsection{Validation}

We first introduce the parameters used in the discretization. As mentioned in $\S 2.2 .1$, $\alpha_{\text {cut }}=a^{-1}=1$ and $R_{\text {cut }}=4 \alpha_{\text {cut }}^{-1}$ are adopted following the recommendation in Pranay et al. (2010). Cubic spectral elements of size 1 with $5 \times 5 \times 5$ GLL points are used to discretize the fluid domain, where the mean grid spacing $h_{\text {mean }}=1 / 4$ well satisfies the relation $\alpha_{\text {cut }} h_{\text {mean }} \leqslant 0.5$ proposed in Kumar \& Graham (2012). Rigorous tests are carried out to be sure that the results are independent of the mesh resolution and the cut-off radius $R_{\text {cut }}$, supporting the current choice. For the membrane dynamics, $N_{S H}=$ 24 modes with a dealiasing factor $M_{S H} / N_{S H}=2$ are chosen to exploit the geometrical symmetry.

The tank-treading motion of an initially spherical capsule in homogeneous shear flow is selected as the first validation case of our implementation. The capsule evolves into a prolate and reaches a steady deformed shape where the membrane continuously rotates in a tank-treading fashion. The time-dependent capsule deformation is measured by the Taylor parameter

$$
D=\frac{L_{\max }-L_{\min }}{L_{\max }+L_{\min }}
$$

where $L_{\max }$ and $L_{\min }$ are the maximum and minimum dimensions of the capsule in the shear plane. We display $D$ as a function of time for neo-Hookean capsules with a varying $\mathrm{Ca}$ and no bending stiffness in figure 2(a). Good agreement is observed between our simulations and those of Pranay et al. (2010).

We next compare cases including bending modulus against the results of Pozrikidis (2001) and Le (2010), see figure 2(b). The agreement is generally good although small differences appear when the capsule reaches its equilibrium shape. This is probably due to the different discretization used to evaluate the high-order derivatives for the calculation of bending moments. As pointed out by Pozrikidis (2001), his simulations suffer from 'significant inaccuracies' at high capsule deformations; our results agree very well with theirs in the small deformation regime (around $t<0.5$ ). To verify the nearly singular integration, we therefore also simulate a capsule with zero bending 


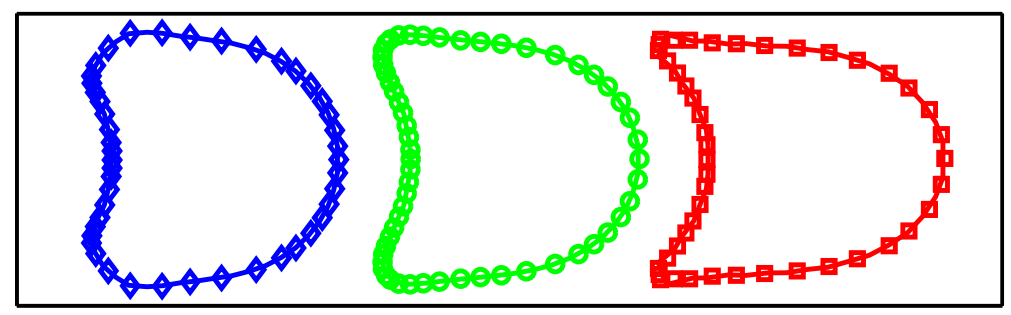

FIGURE 3. (Colour online) Equilibrium profiles of neo-Hookean capsules with different capillary number $C a$ in a square duct of size $l_{\text {duct }}$ and confinement $2 a / l_{\text {duct }}=0.9, C b=$ 0 . The symbols correspond to the results of $\mathrm{Hu}$ et al. (2011) and the solid lines to our simulations using $N_{S H}=24$ modes to represent the membrane surface: $\diamond, C a=0.02 ; \bigcirc$, $C a=0.05 ; \square, C a=0.10$.

stiffness compressed in a confined square duct, and report excellent agreement with the data of $\mathrm{Hu}$ et al. (2011), see figure 3.

\section{Results}

We consider an initially spherical capsule located at the centre of the square duct, as deformable objects tend to move towards the centreline due to the Fåhraeus effect. We impose the analytical velocity profile of a rectangular duct flow (Spiga \& Morino 1994) at the inlet with mean velocity $V_{C}$. We anchor the centre of the capsule at $(0,-5,0) a$, i.e. $5 a$ away from both the computational inlet and the corner, and release it after it has reached its equilibrium shape. This distance is large enough for the interaction between the capsule and the inlet/corner to be negligible during this initial phase.

We investigate the influence of the capillary number $C a$ on the dynamics of the capsule, including its deformation, trajectory, velocity, surface area and principal tensions. The reduced bending modulus is fixed to $C b=0.04$, unless otherwise specified. In addition, we examine the influence of the confinement and of the geometry of the corner.

We note that $C b \approx 0.01$ for RBCs, according to Pozrikidis $(2005 a)$ and Zhao et al. (2010). We adopt the larger value $C b=0.04$ to prevent the bulking of membrane that would easily destabilize the simulations. Luckily, we found the influence of varying $C b$ is much weaker than that of varying the capillary number $C a$; $C b$ represents the relative strength of bending over shearing and its variation from 0.01 to 0.04 accounts for only $3 \%$ of the shear modulus.

\subsection{Square duct flow with a $90^{\circ}$ straight corner}

We begin by investigating the motion of a capsule transported in a moderately confined square duct (of width $H_{x}=3 a$ ) with a straight corner. Throughout the work, the cross-section of the vertical and horizontal duct remains the same, $H_{y} \equiv H_{x}$.

The background flow in the absence of capsules is referred to as the single-phase flow and is illustrated in figure 4 in the $x-y$ plane. We show five trajectories $(S 1-S 5)$ starting from equally spaced points on the line $y=-9 a, x \in[-1.2,1.2] a$; they are ordered from the outer to the inner corner so that $S 3$ goes through the centre of the domain. The velocity magnitude $V_{S}(t)$ is symmetric about $t=0$ : when the minimum is reached for $S 1-S 3$, a maximum occurs for $S 4$ and $S 5$. 


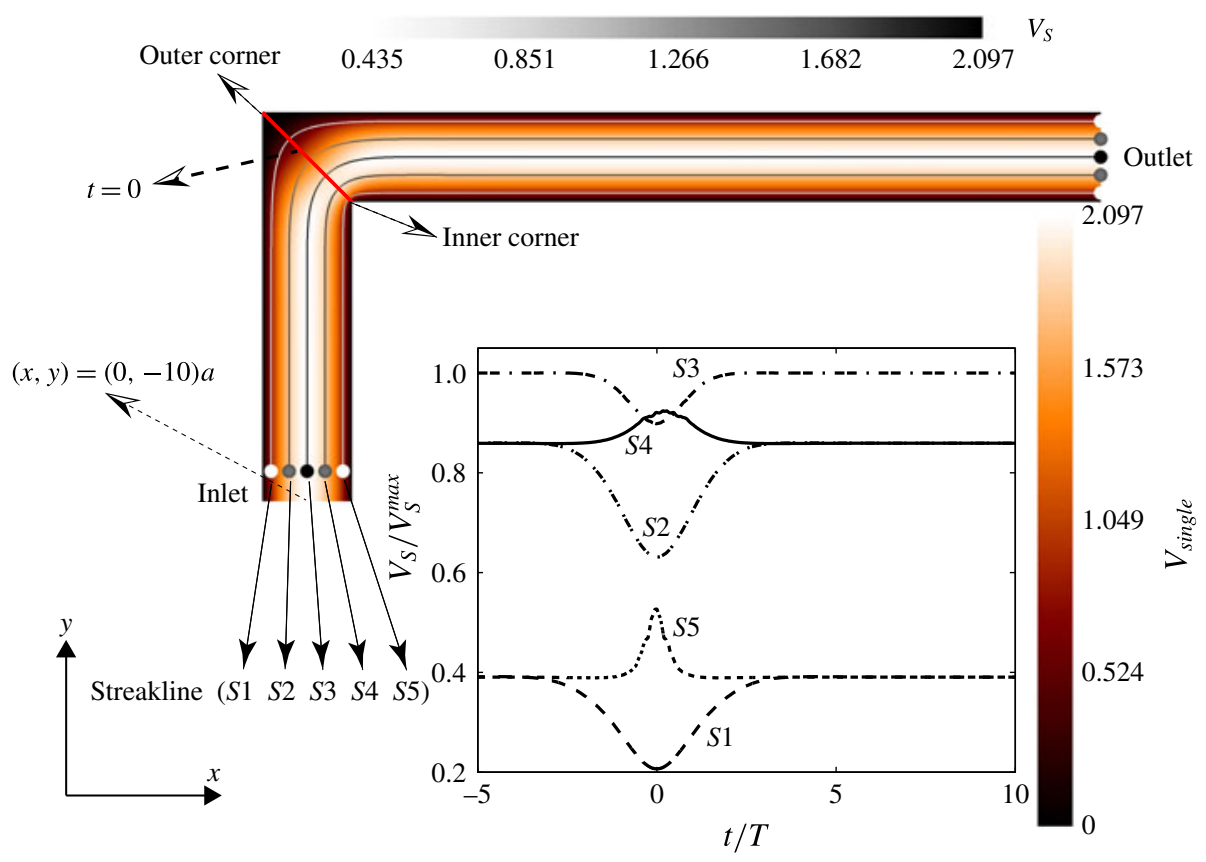

FIgURE 4. (Colour online) The velocity field pertaining the single-phase flow in a square duct of width $H_{x}=3 a$ with a straight corner. The flow field and streaklines are coloured by their magnitude $V_{\text {single }}$ and $V_{S}$ respectively. The streaklines $(S 1-S 5)$ start from points equally spaced between $(-1.2,-9) a$ and $(1.2,-9) a$. $V_{S}$ divided by the maximum flow velocity is depicted in the inset versus time, where $t=0$ corresponds to the time when a fluid particle crosses the corner symmetry axis.
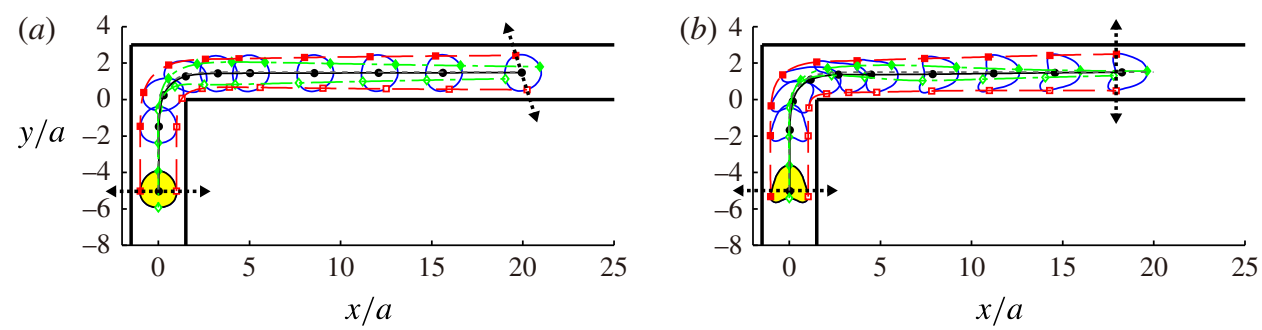

Figure 5. (Colour online) Trajectories and profiles on the $x-y$ plane of capsules with capillary number ( $a$ ) $C a=0.075$ and $(b) C a=0.35$, reduced bending modulus $C b=$ 0.04 and confinement $H_{x} / a=3$. The shading denotes the initial equilibrium shape. The curve with circles represents the centroid path. The dash-dotted curve is the centreline streakline of the single-phase flow. Dash-dotted curves with filled and hollow diamonds show trajectories of front and rear apices, respectively; dashed curves with squares show that of left and right apices. The dashed arrows connecting the left and right apices indicate the rotation of the membrane.

\subsubsection{Trajectory of the capsule and membrane rotation}

The deformation and trajectories of capsules with $C a=0.075$ and $C a=0.35$ are displayed in figures $5(a)$ and $5(b)$, respectively. The centroid trajectory (curve 
(a)

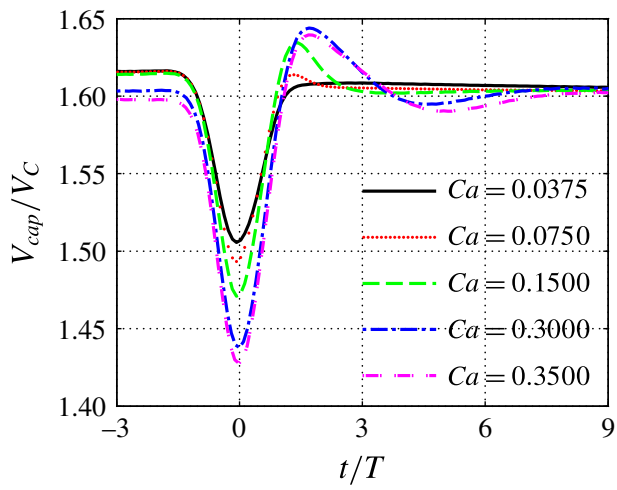

(b)

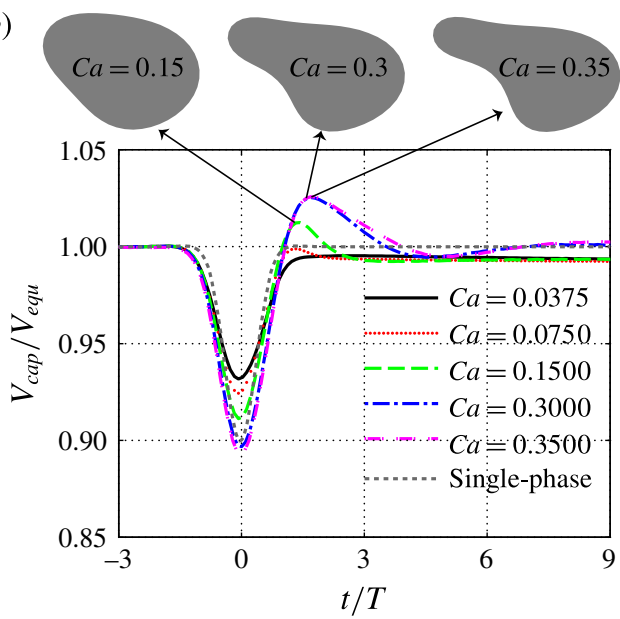

FIgURE 6. (Colour online) Time evolution of the velocity of the capsule centre, $V_{\text {cap }}$, scaled by the mean velocity $V_{C}$ of the duct in $(a)$, and by the cell velocity at equilibrium $V_{e q u}$ in $(b)$. The confinement $H_{x} / a=3$ and results are shown for capsules with $C a=0.0375$, $0.075,0.15,0.3$ and 0.35 and a reduced bending modulus $C b=0.04$. The shape of the capsules at the maximum velocity is provided in $(b)$.

with circles) closely matches the middle streakline $S 3$ (dash-dotted curve) and is almost insensitive to the membrane elasticity. We also mark and trace the four apices of the capsule from the equilibrium shape. For $C a=0.075$, we identify a clear rotation by comparing the initial and final positions of the apices. The front and rear apices initially on $S 3$ follow trajectories (indicated by filled and hollow diamonds respectively) deviating from $S 3$ significantly; the front/rear apex drifts towards the outer/inner corner, eventually remaining above/below the centroid trajectory. The left/right apex starts from the same vertical position and approximately moves along the streakline $S 1 / S 5$. These are characterized by a decreasing/increasing velocity around the corner (see figure 4); as a result, the right apex travels beyond the left, as shown in figure 5. The material points on the capsule rotate, therefore, in the anti-clockwise direction. This rotation is induced by the flow near the corner: this is spatially non-uniform across the duct and the material points near the inner/outer corner are advected by the accelerating/decelerating flow, which results in a net membrane rotation. In the case of $C a=0.35$, the membrane rotation is not as clear. Compared to the case with $C a=0.075$, the right apex is closer to the wall where the underlying flow is slower, thus compensating the increase of the fluid velocity near the corner. Hence, the left and right apices are roughly found at the same streamwise location downstream of the corner.

\subsection{Velocity of the capsule}

The velocity of the capsule centre, $V_{\text {cap }}$, scaled by the mean velocity $V_{C}$ is reported in figure $6(a)$ as a function of time; the same quantity instead divided by the equilibrium velocity $V_{\text {equ }}$, is depicted in figure $6(b)$, together with the velocity on the centre streakline $S 3$ of the single-phase flow (cf. figure 4). All capsules move faster than the average flow velocity, a signature of the Fåhraeus effect. Note that the equilibrium velocity $V_{\text {equ }}$, the velocity in a straight duct, decreases slightly with $\mathrm{Ca}$. 
This was also discussed by Kuriakose \& Dimitrakopoulos (2011) (figure $8 a$ in their paper): $V_{\text {equ }}$ increases with $\mathrm{Ca}$ as $H_{x} / a=2.5$ but decreases in the less confined case, $H_{x} / a=10 / 3$; our simulations with $H_{x} / a=3$ are between the two cases in Kuriakose \& Dimitrakopoulos (2011) and confirm the negative trend of $V_{\text {equ }}$ at low confinement. The velocity of the capsule is related to the thickness of the capsule-wall lubrication film; a thinner film induces higher viscous dissipation and thus reduces the capsule velocity; the thickness of the film when the capsule is slower $(\mathrm{Ca}=0.35)$, is about $93 \%$ that of the fast capsule $(\mathrm{Ca}=0.0375)$.

The velocity of the capsule decreases when approaching the corner and increases when leaving it, reflecting the behaviour of the background flow; the time histories reveal a minimum located at $t=0$, when the particle centre is on the corner axis. This minimum velocity decreases with the capillary number $\mathrm{Ca}$; a slightly thinner lubrication film is observed at the corner axis as $\mathrm{Ca}$ changes from 0.35 to 0.075 (see figure 5). Unlike the underlying flow, the motion of the capsule clearly breaks the time-reversal symmetry about $t=0$, revealing an overshoot during the recovery stage; this symmetry breaking becomes more evident for higher $\mathrm{Ca}$. This loss of symmetry is related to the viscoelasticity induced by the fluid-capsule interaction.

We show the shape of the capsule associated with the larger velocity overshoots $(C a=0.15,0.3$ and 0.35$)$ in figure $6(b)$ at the time the peak velocity is attained. A clear tail-like protrusion is observed for the two largest $\mathrm{Ca}$, due to the streamwise stretching induced by the background accelerating flow. Such a shape is responsible for the observed velocity overshoot, as the streamwise membrane extension corresponds to a decrease of the cross-flow extension of the capsule (its volume must be conserved). This causes a larger distance between capsule and wall and a reduced viscous dissipation. Not surprisingly, as the capsule leaves the corner, its vertical dimension recovers to the equilibrium value and so does the velocity.

The velocity does not converge exactly to its equilibrium value: a maximum relative difference of around $0.6 \%$ is observed. It would require a prohibitively long computational domain and integration time to obtain a precise convergence as also observed by Woolfenden \& Blyth (2011); the physics of the final capsule relaxation is therefore beyond the scope of the present investigation.

\subsection{Capsule surface area and deformation}

The capsule surface area, $A$, is used as indicator of the global deformation. This is reported in figure 7 for the same cases as in figure 6. When the capsule is far away from the corner, the area maintains the equilibrium value $A_{\text {equ }}$, an increasing function of $C a$. The area variation $A_{\text {equ }} / 4 \pi a^{2}-1$ is almost zero for the cases with $C a=0.0375$ and 0.075 , whereas it grows to values around 0.1 when $C a=0.35$. As the capsule travels around the corner, the deformation reaches its peak value and its variation $A_{\text {peak }} / 4 \pi a^{2}-1$ is around 0.2 for the highest $C a$ investigated.

Ideally, the dependence of the area on the capillary number can be used to deduce the membrane properties of capsules, as shown by Lefebvre et al. (2008), Chu et al. (2011) and $\mathrm{Hu}$ et al. (2013), who focus on the identification based on deformation. However, direct measurement of the total surface area is not easy, while it is more feasible to measure its two-dimensional projection. As a consequence, we display the projection of the capsule area on the $x-y$ mid-plane in figure $7(b)$.

The projected area $A_{x y}$ varies with time and cell deformability in a more complicated way. For the two smallest values of $\mathrm{Ca}, A_{x y}$ reaches the minimum around $t=0$ before recovering to the equilibrium value past the corner. The cases 

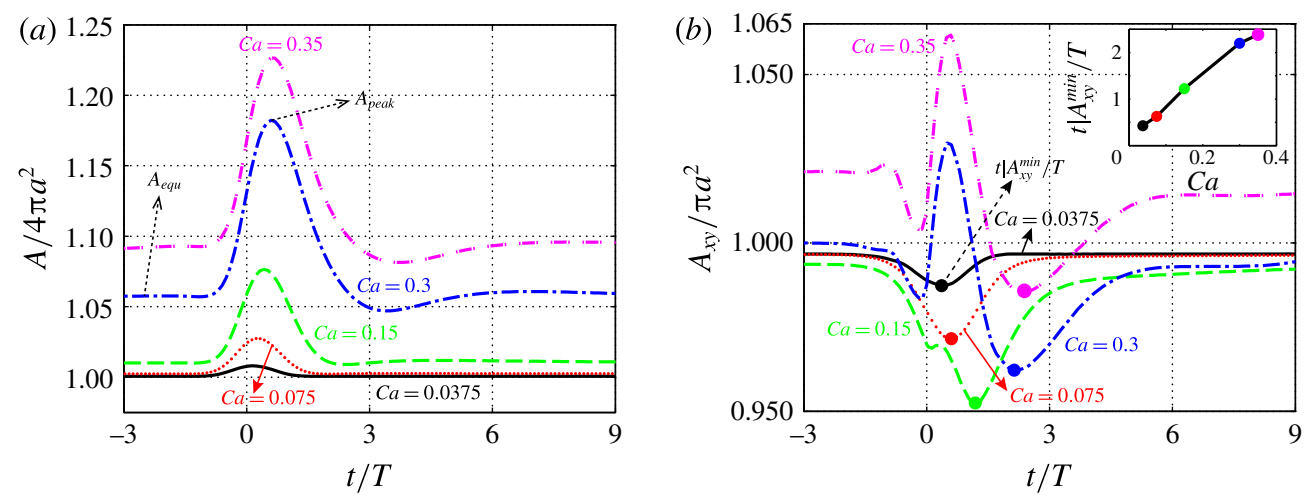

FIgURE 7. (Colour online) Time evolution of the non-dimensional surface area for the same capsules in figure 6. (a) Total surface area $A / 4 \pi a^{2}$ and $(b)$ projected area on the $x-y$ plane $\left.A_{x y} / \pi a^{2}\right) ; A_{\text {equ }}$ and $A_{\text {peak }}$ indicate the equilibrium and peak value of $A$ respectively. Solid circles indicate the time $t \mid A_{x y}^{\min } / T$ when the minimum area $A_{x y}$ is achieved. The inset of (b) shows $t \mid A_{x y}^{\min } / T$ versus $C a$.

characterized by $C a=0.3$ and 0.35 display a clear peak in deformation right after $t=0$, with two sharp troughs, one before and one after. This wavy variation is already visible when $C a=0.15$, although weak. Further examination of the behaviour in the range $C a \in[0.15,0.3]$ confirms that the time traces of the area deformation become more wavy as $\mathrm{Ca}$ increases; more elastic material is prone to exhibit more oscillatory motions under the same excitation, the spatially developing flow here. The inset of figure $7(b)$ shows the time $t \mid A_{x y}^{\min } / T$ corresponding to the minimum projected area $A_{x y}$. This can be regarded as the phase lag of the capsule and it increases almost linearly with $C a$.

\subsection{Principal tension on the capsule}

The tension developing on the membrane is of great importance since it influences the release of molecules (Goldsmith et al. 1995) and ATP (Wan, Ristenpart \& Stone 2008) by RBCs and causes haemolysis, to cite two examples. We analyse the principal tension $\tau_{i}^{P}(i=1,2)$, to better understand the potential mechanical damage of capsules passing through a corner. For any definition of strain energy function $W_{S}\left(I_{1}, I_{2}\right)$, the $\tau_{i}^{P}$ are derived as (Skalak et al. 1973):

$$
\begin{aligned}
\tau_{1}^{P} & =2 \frac{\lambda_{1}}{\lambda_{2}}\left(\frac{\partial W_{S}}{\partial I_{1}}+\lambda_{2}^{2} \frac{\partial W_{S}}{\partial I_{2}}\right), \\
\tau_{2}^{P} & =2 \frac{\lambda_{2}}{\lambda_{1}}\left(\frac{\partial W_{S}}{\partial I_{1}}+\lambda_{1}^{2} \frac{\partial W_{S}}{\partial I_{2}}\right) .
\end{aligned}
$$

We consider the major principal tension: $\max \left(\tau_{1}^{P}(\boldsymbol{x}, t), \tau_{2}^{P}(\boldsymbol{x}, t)\right)$ and the isotropic principal tension $\left(\tau_{1}^{P}(\boldsymbol{x}, t)+\tau_{2}^{P}(\boldsymbol{x}, t)\right) / 2$; their surface maxima $\tau_{\text {max }}^{P}(t)$ and $\tau_{\text {max }}^{P_{I S O}}(t)$ are defined as

$$
\begin{gathered}
\tau_{\text {max }}^{P}(t)=\max _{\boldsymbol{x}, i=1,2}\left(\tau_{i}^{P}(\boldsymbol{x}, t)\right), \\
\tau_{\max }^{P_{\text {ISO }}}(t)=\max _{\boldsymbol{x}}\left(\left(\tau_{1}^{P}(\boldsymbol{x}, t)+\tau_{2}^{P}(\boldsymbol{x}, t)\right) / 2\right),
\end{gathered}
$$

where $(t)$ will be omitted hereinafter for the sake of clarity. 


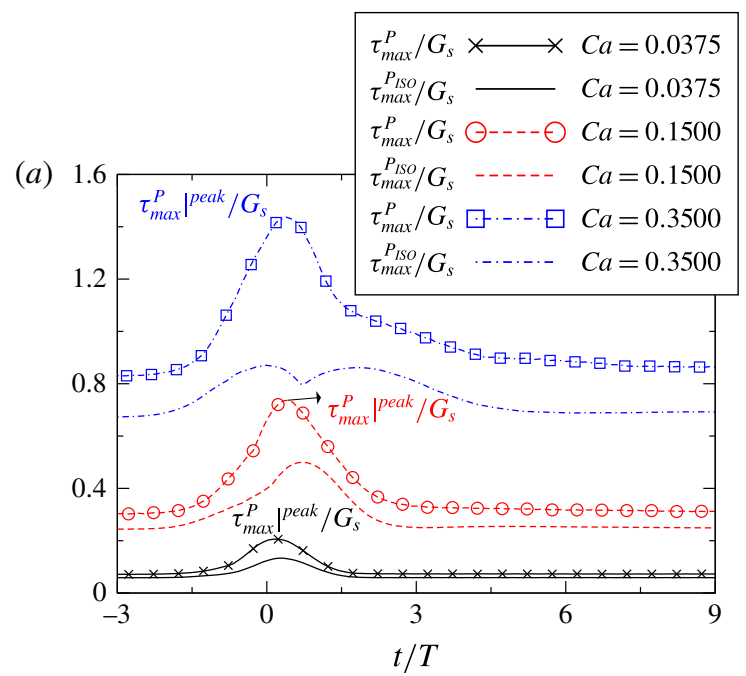

(b)

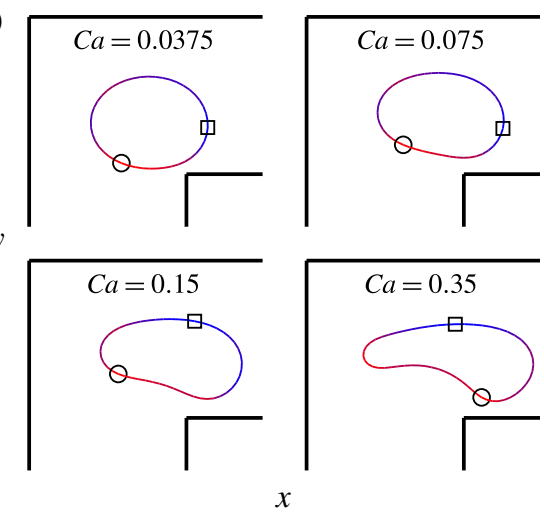

FIgURE 8. (Colour online) (a) Time evolution of the maximum of the two principal tensions in the non-dimensional form, $\tau_{\max }^{P} / G_{s}$ and $\tau_{\max }^{P_{\text {ISO }}} / G_{s}$, for the major and isotropic principal tension respectively. (b) Position and contour of the capsules on the $x-y$ plane when reaching the maximum major principal tension $\left.\tau_{\max }^{P}\right|^{\text {peak }}$. The magnitude of $\tau_{\max }^{P}$ is indicated by light grey/dark grey (red and blue online) for low/high values and its minimum/maximum position by the circle/square.

The temporal evolution of $\tau_{\max }^{P} / G_{s}$ and $\tau_{\max }^{P_{I S O}} / G_{s}$ is shown in figure 8 for capsules going through a straight corner. For most cases, both quantities increase monotonically with $\mathrm{Ca}$, reaching the peak values slightly after the corner before relaxing back to the equilibrium value. The difference between the two tensions is more pronounced at the corner, $\tau_{\max }^{P} / \tau_{\max }^{P_{I S O}} \approx 2$, and weak in the straight ducts. We also show in the figure the shape of some capsules when the maximum major principal tension is reached, with the minimum and maximum of $\tau_{\max }^{P}$ indicated by a circle and square respectively. The maximum of $\tau_{\max }^{P}$ develops at the front for the capsules with $C a=0.0375,0.075$, while it moves to the top part for $\mathrm{Ca}=0.35$. Material points are prone to accumulate at the rear of the capsule and the principal tension is therefore relatively low. As $\mathrm{Ca}$ increases, the rear part of the capsule changes from a convex to concave shape, something more evident for the case $C a=0.35$.

\subsection{The influence of confinement and geometry of the corner}

We examine first the influence of confinement on the capsule motion by varying the width $H_{x} / a$ from 2.7 to 4 . The velocity of the capsule, divided by its equilibrium velocity, is shown in figure 9 for $C a=0.15$. The time-symmetry around $t=0$ is almost preserved for the least confined case $H_{x} / a=4$. As the confinement increases, the symmetry breaking discussed before and the corresponding velocity overshoot become more apparent. These are associated with a decrease of the minimum velocity at the corner. As $H_{x} / a$ varies from 3.5 to 2.7 , the velocity overshoot also clearly increases. The shape of the capsule at the time of maximum velocity is also displayed in the figure. The capsule with highest velocity is more elongated and has a larger distance from the wall (lower lubrication friction), in analogy to the observations in $\S 3.2$ for capsules of different elasticity. 

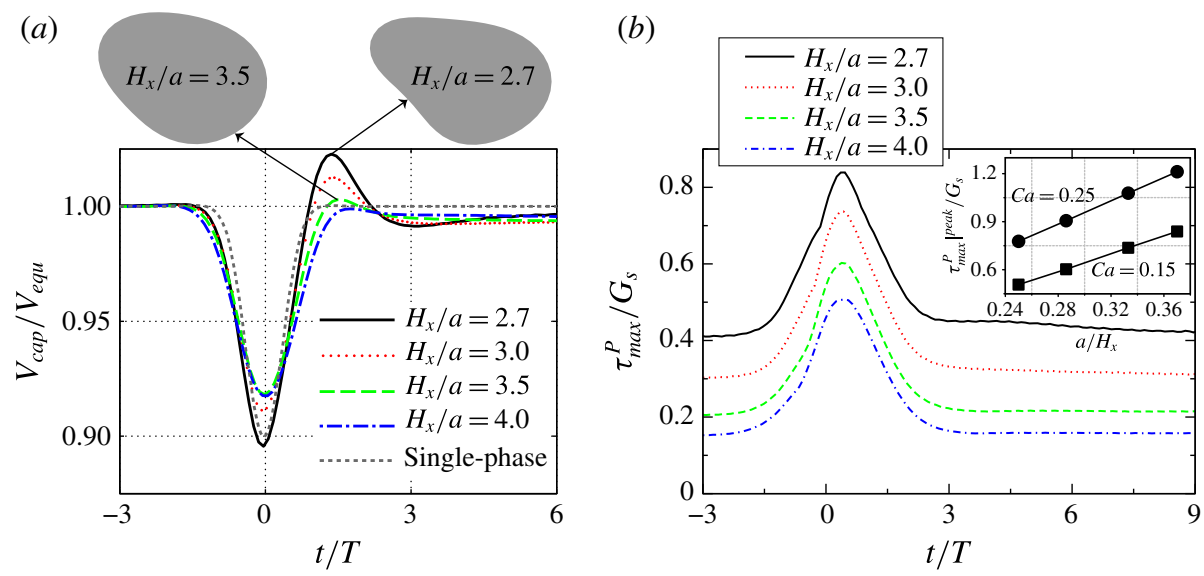

FIgURE 9. (Colour online) Time evolution of $(a)$ the velocity $V_{c a p} / V_{\text {equ }}$ and $(b)$ the major principal tension $\tau_{\max }^{P} / G_{s}$ for $C a=0.15$ where the width of the square duct is varied from $H_{x} / a=2.7$ to $H_{x} / a=4$. The shape of the capsule at the time when the maximum velocity is attained is also given in $(a)$. The inset of $(b)$ shows the relation between the maximum major principal tension $\left.\tau_{\text {max }}^{P}\right|^{\text {peak }} / G_{s}$ and the inverse of the duct width $a / H_{x}$ for $C a=0.15$ and 0.25 .

The surface maximum of the non-dimensional major principal tension $\tau_{\max }^{P} / G_{s}$ is depicted in figure $9(b)$ for the same cases: $\tau_{\max }^{P}$ increases monotonically with the confinement. The maximum over time of $\left.\tau_{\text {max }}^{P}\right|^{\text {peak }} / G_{s}$ is displayed versus $a / H_{x}$ for $C a=0.15$ and 0.25 in the inset of the same figure to show that the peak principal tension increases linearly with $a / H_{x}$. This relationship may be useful to estimate the mechanical stress on/damage to the cells in micro-fluidic devices during the design stage.

Finally, we consider a curved corner with inner radius $R_{c} / a=1$. In figure 10 we compare the principal tensions on the membrane with those in the straight corner for capillary numbers $C a=0.075$ and 0.3 . Except for the isotropic principal tension of the capsule with $C a=0.075$, the principal tension decreases significantly in the curved corner.

\section{Discussion and conclusion}

We investigate the motion of a three-dimensional deformable capsule, whose membrane obeys the neo-Hookean constitutive relation, in a square duct flow with a corner. We present in this work a new implementation of the BIM accelerated by the GGEM, the general-geometry Ewald method, to resolve fluid-structure interactions at low Reynolds number in complex geometries. The algorithm is coupled with a spectral method based on spherical harmonics for the membrane dynamics. In this section, we first discuss the details of the numerical method, followed by a short summary of the main physical findings.

The GGEM shares similarities with the classic immersed boundary methods (IBM) (Mittal \& Iaccarino 2005). Both approaches require a Lagrangian mesh for the suspended objects and an Eulerian (typically Cartesian) mesh for the fluid; the Dirac delta function, representing the localized forcing from the object, is approximated numerically. In the IBM, the localized force is spread from each Lagrangian point onto a number of surrounding Eulerian points to enforce the desired boundary conditions 


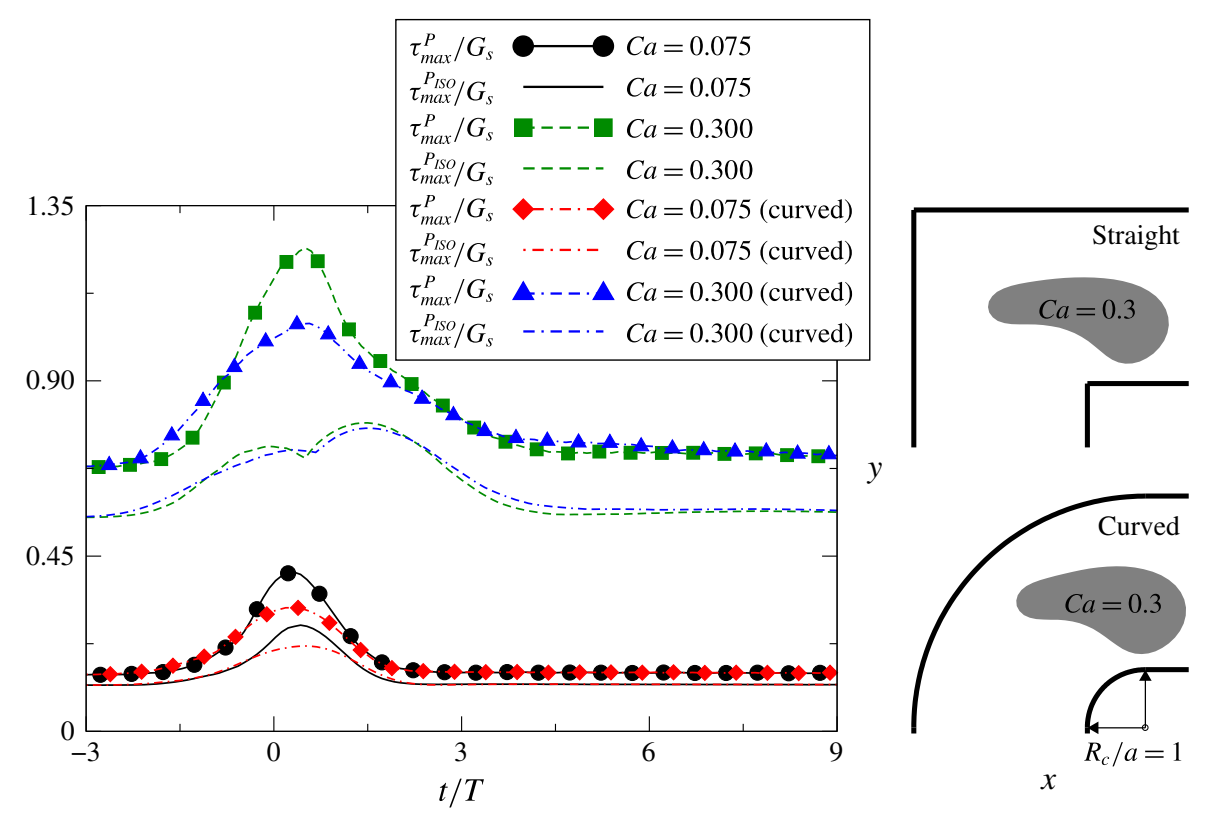

FIgURE 10. (Colour online) Time evolution of the major principal tension $\tau_{\max }^{P} / G_{s}$ for capsules $C a=0.075 / 0.3$ through a straight and a curved corner. The curvature radius of the curved corner is $R_{c} / a=1$. The shape of capsule on the $x-y$ plane is shown when it reaches the peak $\tau_{\max }^{P}$.

at the fluid/solid interface. The accuracy of IBM degenerates if close hydrodynamic interactions arise, which may require ad hoc corrections to account for the correct lubrication forces (Lashgari et al. 2014). As shown in (2.5), the Dirac delta function is also smeared in the local problem of GGEM, but its singular behaviour can be solved accurately by boundary integral techniques with singular integration. If a regularized-Stokeslet technique is instead employed as in Pranay et al. (2010) and Hernández-Ortiz et al. (2007), the GGEM closely resembles an IBM as proved by Pranay et al. (2010). Note also that a traditional IBM requires a uniform Eulerian grid to conserve the moments of the force and sophisticated treatments are needed to adapt IBM to a non-uniform and/or unstructured grid as done by Pinelli et al. (2010) and Mendez, Gibaud \& Nicoud (2014) among others. One advantage of the GGEM is that the smoothing of the local forcing is exactly compensated by the global forcing due to the linearity of Stokes equations. The integral of the force field and its moments are therefore preserved. Hence, Stokes solvers based on uniform or non-uniform grids can be readily coupled to the GGEM. In our case, the Eulerian grid points (GLL points) are non-uniformly distributed as shown in figure 1 .

GGEM was originally designed to resolve the hydrodynamic interaction among multiple particles in Stokes flows. Suppose that there are $N^{p}$ particles and each of them is discretized into $M$ Lagrangian points, then the total number of points is $N^{p} M$ and the number of degrees of freedom $N_{d} \sim N^{p} M$. For traditional non-accelerated BIM, the number of operations required to form the mobility matrix scales with $N_{d}^{2}$. This poses the major difficulty in applying BIM to a large number of particles. Accelerating techniques for BIM have thus been developed to overcome this restriction, and GGEM is one of them. The decomposition of the Dirac delta function into two parts reduces the number of operations from $O\left(N_{d}^{2}\right)$ to $O\left(N_{d}\right)$ or $O\left(N_{d} \log N_{d}\right)$ 
(Hernández-Ortiz et al. 2007). The modified Green's function for the local problem is designed such that the local solution decays exponentially over a distance of about $\alpha_{c u t}^{-1}$. Neglecting interactions that occur beyond the cut-off distance $R_{c u t} \sim \alpha_{c u t}^{-1}$, the number of operations for the local solution decreases and scales linearly with $N_{d}$. The scaling of the global problem depends on the mesh-based solver, as well as the geometry and boundary conditions of the computational domain. The solver used here, NEK5000, is computationally more expensive than Fourier-based methods such as those used in Kumar \& Graham (2012), but it allows for arbitrary geometries and is highly parallel.

It is hard to provide a scaling for the global part of the problem in general geometries; however, the advantage of a numerical approach like that pursued here relies on two points: (i) GGEM provides a convenient way to reshape the $O\left(N_{d}^{2}\right)$ long-ranged interactions and pack them into a problem solvable by a mesh-based solver; (ii) a highly parallel solver is chosen to considerably reduce the computational time. Note that a naive parallelization of traditional BIM implementations is not possible for a large $N_{d}$ due to the prohibitively large amount of memory needed and poor scalability of the linear system with a dense matrix. It should be noted that our implementation might be less efficient than traditional BIM for studying the dynamics of one (as we do here) or a few capsules. This is, however, our first step in the development of a computational framework for suspensions of deformable/rigid particles in general geometries.

This numerical approach is used here to examine the motion of a capsule through a square duct with a corner, focusing on its trajectory, velocity, deformation, total and projected surface area, and principal tension. We aim to better understand the transient dynamics of capsules in a micro-fluidic device with realistic geometries.

We study the deformation of the capsules when varying the capillary number, the ratio of viscous to elastic forces. The capsule trajectories closely follow the underlying flow and are therefore rather insensitive to how the capsules deform. Due to the strong confinement, deviations from the underlying streamline require a significant viscous dissipation. Conversely, the deformability of a capsule closely influences its shape, velocity and the mechanical stress developing on the membrane as documented in the results section.

The corner flow can be potentially adopted to infer the material properties of deformable particles as shown by Lefebvre et al. (2008) and Chu et al. (2011) using straight tube or channel flows. Unlike these works, transient effects are present in the flow past a corner because of its spatial inhomogeneity. When the capsule is far away from the corner, the surface area, velocity and principal tension reach equilibrium values that are a function of the capillary number $C a$. When flowing around the corner, the membrane area and tension reach their maxima while the velocity is at its minimum; these extrema are shown here to clearly depend on $\mathrm{Ca}$. By utilizing a spatially developing flow, the shape and/or velocity of the capsules can be measured not only at the equilibrium state but also during the transient motions. More robust and accurate inverse methods may be developed using measurements of the extrema values. We further note that a new time scale is introduced in the corner flow, hence the phase lag of the capsule can be identified as illustrated by the temporal evolution of the projected area (see figure $7 b$ ); this quantity indicating the viscoelasticity of capsule is not accessible from traditional steady flow experiments. The spatially developing flow provides supplemental information characterizing the material properties of natural and synthetic cellular structures.

The capsule shape is also closely linked to its velocity. For low $\mathrm{Ca}$, the velocity is similar to that of the underlying flow, with an almost perfect time-reversal symmetry; 
as $\mathrm{Ca}$ increases, i.e. more pronounced deformations, this symmetry is broken and a velocity overshoot appears past the corner. The streamwise elongation of the capsule increases the capsule-wall distance, and the corresponding lower viscous dissipation can explain the higher capsule velocity.

The surface maxima of the major and isotropic principal tension become significantly different only when the capsule is flowing around the corner. During this time, the maximum major principal tension appears at the front of capsules for configurations with low $\mathrm{Ca}$, and shifts towards the outer edge as $\mathrm{Ca}$ increases.

We have also examined the influence of confinement and of the geometry of the corner. We identify a positive correlation between the asymmetry of the velocity profile and the level of confinement. The peak of the major principal tension increases linearly with the inverse of the duct width $a / H_{x}$. Finally, we show that a curved corner reduces the major principal tension and the deformation of the capsule. We believe the present work can improve our understanding of the capsule motion in complex geometries and support the design of micro-fluidic devices with multiple corners and branches.

\section{Acknowledgements}

We thank Professors D. Barthès-Biesel, M. D. Graham and J. B. Freund and Dr H. Zhao for useful discussions. Funding by VR (the Swedish Research Council), the Linné FLOW Centre at KTH and computer time via SNIC (Swedish National Infrastructure for Computing) and HPC at EPFL are greatly acknowledged. L.Z. acknowledges the financial support from the European Research Council (ERC) grant 'simcomics-280117' as a postdoc researcher at EPFL where part of the work is performed. This research is also supported by the ERC grant '2013-CoG-616186, TRITOS' to L.B.

\section{REFERENCES}

Adams, J. C. \& Swarztrauber, P. N. 1997 Spherepack 2.0: a model development facility. NCAR Tech. Note.

Autrusson, N., Guglielmini, L., Lecuyer, S., Rusconi, R. \& Stone, H. A. 2011 The shape of an elastic filament in a two-dimensional corner flow. Phys. Fluids 23, 063602.

Barber, J. O., Alberding, J. P., Restrepo, J. M. \& Secomb, T. W. 2008 Simulated twodimensional red blood cell motion, deformation, and partitioning in microvessel bifurcations. Ann. Biomed. Engng 36 (10), 1690-1698.

BARTHÈs-BIESEL, D. 1980 Motion of a spherical microcapsule freely suspended in a linear shear flow. J. Fluid Mech. 100 (4), 831-853.

BARTHÈS-BIESEL, D. 1981 The time-dependent deformation of a capsule freely suspended in a linear shear flow. J. Fluid Mech. 113, 251-267.

BARthÈs-BIEsel, D. 2011 Modeling the motion of capsules in flow. Curr. Opin. Colloid Interface 16 (1), 3-12.

Braunmüller, S., Schmid, L. \& Franke, T. 2011 Dynamics of red blood cells and vesicles in microchannels of oscillating width. J. Phys.: Condens. Matter 23 (18), 184116.

BRUNO, O. P. \& KUNYANSKY, L. A. 2001 A fast, high-order algorithm for the solution of surface scattering problems: basic implementation, tests, and applications. J. Comput. Phys. 169 (1), $80-110$.

Chu, T. X., Salsac, A.-V., Leclerc, E., Barthès-Biesel, D., Wurtz, H. \& Edwards-Lévy, F. 2011 Comparison between measurements of elasticity and free amino group content of ovalbumin microcapsule membranes: discrimination of the cross-linking degree. J. Colloid Interface Sci. 355 (1), 81-88. 
Danker, G., Vlahovska, P. M. \& Misbah, C. 2009 Vesicles in Poiseuille flow. Phys. Rev. Lett. $102(14), 148102$.

Doddi, S. K. \& BAGCHI, P. 2008 Lateral migration of a capsule in a plane Poiseuille flow in a channel. Intl J. Multiphase Flow 34 (10), 966-986.

Dodson, W. R. \& Dimitrakopoulos, P. 2009 Dynamics of strain-hardening and strain-softening capsules in strong planar extensional flows via an interfacial spectral boundary element algorithm for elastic membranes. J. Fluid Mech. 641, 263-296.

Fischer, P., Lottes, J., Pointer, D. \& Siegel, A. 2008 a Petascale algorithms for reactor hydrodynamics. J. Phys. Conf. Ser. 125, 012076.

Fischer, P. F., Lottes, J. W. \& Kerkemeier, S. G. $2008 b$ nek5000 Web page. http://nek5000.mcs.anl.gov.

FISCHER, T. \& SCHMID-SCHÖNBEIN, H. 1978 Tank tread motion of red cell membranes in viscometric flow: behavior of intracellular and extracellular markers (with film). In Red Cell Rheology, pp. 347-361. Springer.

Foessel, E., Walter, J., SAlsac, A. V. \& Barthès-Biesel, D. 2011 Influence of internal viscosity on the large deformation and buckling of a spherical capsule in a simple shear flow. J. Fluid Mech. 672, 477-486.

Freund, J. B. 2007 Leukocyte margination in a model microvessel. Phys. Fluids 19, 023301.

Freund, J. B. 2014 Numerical simulation of flowing blood cells. Annu. Rev. Fluid Mech. 46 (1), 67-95.

Freund, J. B. \& ZhaO, H. 2010 A high-resolution fast boundary-integral method for multiple interacting blood cells. In Computational Hydrodynamics of Capsules and Biological Cells (ed. C. Pozrikidis), CRC Press.

Gaehtgens, P., Dührssen, C. \& Albrecht, K. H. 1980 Motion, deformation, and interaction of blood cells and plasma during flow through narrow capillary tubes. Blood Cells 6 (4), 799-817.

Goldsmith, H. L. 1971 Red cell motions and wall interactions in tube flow. In Fed. Proc., vol. 30, p. 1578.

Goldsmith, H. L., Bell, D. N., Braovac, S., Steinberg, A. \& McIntosh, F. 1995 Physical and chemical effects of red cells in the shear-induced aggregation of human platelets. Biophys. J. 69 (4), 1584-1595.

Goldsmith, H. L. \& MARLow, J. 1972 Flow behaviour of erythrocytes. I. Rotation and deformation in dilute suspensions. Phil. Trans. R. Soc. Lond. B 182 (1068), 351-384.

Green, A. E. \& AdKins, J. E. 1970 Large Elastic Deformations, 2nd edn. Oxford University Press.

Hanahan, D. \& Weinberg, R. A. 2000 The hallmarks of cancer. Cell 100 (1), 57-70.

Hernández-Ortiz, J. P., de Pablo, J. J. \& Graham, M. D. 2007 Fast computation of manyparticle hydrodynamic and electrostatic interactions in a confined geometry. Phys. Rev. Lett. 98 (14), 140602.

Hu, X.-Q., SAlsac, A.-V. \& BARThÈS-BIesel, D. 2011 Flow of a spherical capsule in a pore with circular or square cross-section. J. Fluid Mech. 705, 176-194.

Hu, X.-Q., SÉvénié, B., SAlsaC, A.-V., LeClerC, E. \& Barthès-Biesel, D. 2013 Characterizing the membrane properties of capsules flowing in a square-section microfluidic channel: effects of the membrane constitutive law. Phys. Rev. E 87 (6), 063008.

HuAng, Q. \& CRUSE, T. A. 1993 Some notes on singular integral techniques in boundary element analysis. Intl J. Numer. Meth. Engng 36, 2643-2659.

Johnston, P. R. \& Elliott, D. 2005 A sinh transformation for evaluating nearly singular boundary element integrals. Intl J. Numer. Meth. Engng 62 (4), 564-578.

Kaoui, B., Biros, G. \& Misbah, C. 2009 Why do red blood cells have asymmetric shapes even in a symmetric flow? Phys. Rev. Lett. 103 (18), 188101.

Kessler, S., Finken, R. \& Seifert, U. 2008 Swinging and tumbling of elastic capsules in shear flow. J. Fluid Mech. 605, 207-226.

Kumar, A. \& Graham, M. D. 2011 Segregation by membrane rigidity in flowing binary suspensions of elastic capsules. Phys. Rev. E 84 (6), 066316. 
Kumar, A. \& Graham, M. D. 2012 Accelerated boundary integral method for multiphase flow in non-periodic geometries. J. Comput. Phys. 231, 6682-6713.

Kuriakose, S. \& Dimitrakopoulos, P. 2011 Motion of an elastic capsule in a square microfluidic channel. Phys. Rev. E 84 (1), 011906.

Kuriakose, S. \& Dimitrakopoulos, P. 2013 Deformation of an elastic capsule in a rectangular microfluidic channel. Soft Matt. 9 (16), 4284-4296.

Lac, E., Morel, A. \& BArthès-Biesel, D. 2007 Hydrodynamic interaction between two identical capsules in simple shear flow. J. Fluid Mech. 573, 149-169.

Lashgari, I., Picano, F., Breugem, W.-P. \& Brandt, L. 2014 Laminar, turbulent, and inertial shear-thickening regimes in channel flow of neutrally buoyant particle suspensions. Phys. Rev. Lett. 113, 254502.

Lauga, E., Stroock, A. D. \& Stone, H. A. 2004 Three-dimensional flows in slowly varying planar geometries. Phys. Fluids 16, 3051-3062.

LE, D. V. 2010 Effect of bending stiffness on the deformation of liquid capsules enclosed by thin shells in shear flow. Phys. Rev. E 82 (1), 016318.

Lefebvre, Y., Leclerc, E., Barthès-Biesel, D., Walter, J. \& Edwards-Lévy, F. 2008 Flow of artificial microcapsules in microfluidic channels: a method for determining the elastic properties of the membrane. Phys. Fluids 20, 123102.

Lei, H., Fedosov, D., Caswell, B. \& Karniadakis, G. 2013 Blood flow in small tubes: quantifying the transition to the non-continuum regime. J. Fluid Mech. 722, 214-239.

LI, X. \& SARKAR, K. 2008 Front tracking simulation of deformation and buckling instability of a liquid capsule enclosed by an elastic membrane. J. Comput. Phys. 227 (10), 4998-5018.

MadAY, Y. \& PATERA, A. T. 1989 Spectral element methods for the incompressible Navier-Stokes equations. In State-of-the-Art Surveys on Computational Mechanics (A90-47176 21-64), pp. 71-143. American Society of Mechanical Engineers, New York, Research supported by DARPA, vol. 1, pp. 71-143.

Mendez, S., Gibaud, E. \& Nicoud, F. 2014 An unstructured solver for simulations of deformable particles in flows at arbitrary Reynolds numbers. J. Comput. Phys. 256, 465-483.

Misbah, C. 2006 Vacillating breathing and tumbling of vesicles under shear flow. Phys. Rev. Lett. 96, 028104.

Mittal, R. \& IAcCARino, G. 2005 Immersed boundary methods. Annu. Rev. Fluid Mech. 37, 239-261.

Noguchi, H., Gompper, G., Schmid, L., Wixforth, A.\& Franke, T. 2010 Dynamics of fluid vesicles in flow through structured microchannels. Europhys. Lett. 89 (2), 28002.

ÖzkayA, A. N. 1986 Viscous Flow of Particles in Tubes: Lubrication Theory and Finite Element Models. Columbia University.

PARK, S. Y. \& Dimitrakopoulos, P. 2013 Transient dynamics of an elastic capsule in a microfluidic constriction. Soft Matt. 9 (37), 8844-8855.

Pinelli, A., Naqavi, I., Piomelli, U. \& Favier, J. 2010 Immersed-boundary methods for general finite-difference and finite-volume Navier-Stokes solvers. J. Comput. Phys. 229 (24), 9073-9091.

PozRIKIDIS, C. 1995 Finite deformation of liquid capsules enclosed by elastic membranes in simple shear flow. J. Fluid Mech. 297, 123-152.

PozRIKIDIS, C. 2001 Effect of membrane bending stiffness on the deformation of capsules in simple shear flow. J. Fluid Mech. 440, 269-291.

PozRIKIDIS, C. 2003 Numerical simulation of the flow-induced deformation of red blood cells. Ann. Biomed. Engng 31 (10), 1194-1205.

PozRikidis, C. 2005a Axisymmetric motion of a file of red blood cells through capillaries. Phys. Fluids 17 (3), 031503.

PozRikidis, C. $2005 b$ Numerical simulation of cell motion in tube flow. Ann. Biomed. Engng 33 (2), $165-178$.

Pranay, P., Anekal, S. G., Hernandez-Ortiz, J. P. \& Graham, M. D. 2010 Pair collisions of fluid-filled elastic capsules in shear flow: effects of membrane properties and polymer additives. Phys. Fluids 22, 123103. 
Ramanujan, S. \& Pozrikidis, C. 1998 Deformation of liquid capsules enclosed by elastic membranes in simple shear flow: large deformations and the effect of fluid viscosities. $J$. Fluid Mech. 361, 117-143.

Rusconi, R., Lecuyer, S., Guglielmini, L. \& Stone, H. A. 2010 Laminar flow around corners triggers the formation of biofilm streamers. J. R. Soc. Interface 7 (50), 1293-1299.

Schrader, L.-U., Brandt, L., Mavriplis, C. \& Henningson, D. S. 2010 Receptivity to freestream vorticity of flow past a flat plate with elliptic leading edge. J. Fluid Mech. 653, $245-271$.

Secomb, T. W. \& SkALAK, R. 1982 A two-dimensional model for capillary flow of an asymmetric cell. Microvasc. Res. 24 (2), 194-203.

ShI, L., PAN, T. W. \& GlOWINSKI, R. 2012 Lateral migration and equilibrium shape and position of a single red blood cell in bounded poiseuille flows. Phys. Rev. E 86, 056308.

SkAlak, R. \& Branemark, P. I. 1969 Deformation of red blood cells in capillaries. Science 164 (3880), 717-719.

Skalak, R., Tozeren, A., Zarda, R. P. \& Chien, S. 1973 Strain energy function of red blood cell membranes. Biophys. J. 13 (3), 245-264.

SpigA, M. \& Morino, G. L. 1994 A symmetric solution for velocity profile in laminar flow through rectangular ducts. Intl Commun. Heat Mass Transfer 21 (4), 469-475.

Springer, T. A. 1994 Traffic signals for lymphocyte recirculation and leukocyte emigration: the multistep paradigm. Cell 76, 301-314.

Swarztrauber, P. N. \& Spotz, W. F. 2000 Generalized discrete spherical harmonic transforms. J. Comput. Phys. 159 (2), 213-230.

Vlahovska, P. M., Young, Y. N., Danker, G. \& Misbah, C. 2011 Dynamics of a non-spherical microcapsule with incompressible interface in shear flow. J. Fluid Mech. 678, 221-247.

Walter, J., SAlsac, A. V. \& BARthèS-Biesel, D. 2011 Ellipsoidal capsules in simple shear flow: prolate versus oblate initial shapes. J. Fluid Mech. 676, 318-347.

Walter, J., Salsac, A.-V., Barthès-Biesel, D. \& Tallec, P. Le. 2010 Coupling of finite element and boundary integral methods for a capsule in a stokes flow. Intl J. Numer. Meth. Engng 83 (7), 829-850.

Wan, J., Ristenpart, W. \& Stone, H. 2008 Dynamics of shear-induced ATP release from red blood cells. Proc. Natl Acad. Sci. USA 105 (43), 16432-16437.

Woolfenden, H. C. \& Blyth, M. G. 2011 Motion of a two-dimensional elastic capsule in a branching channel flow. J. Fluid Mech. 669, 3-31.

ZARDA, P. R., ChIEN, S. \& SKALAK, R. 1977 Interaction of viscous incompressible fluid with an elastic body. In Computational Methods for Fluid-Solid Interaction Problems (ed. T. Belytschko \& T. L. Geers), pp. 65-82. ASME.

Zhao, H., Isfahani, A. H. G., Olson, L. N. \& Freund, J. B. 2010 A spectral boundary integral method for flowing blood cells. J. Comput. Phys. 229, 3726-3744.

Zhu, L., LAUGA, E. \& BRANDT, L. 2013 Low-Reynolds number swimming in a capillary tube. J. Fluid Mech. 726, 285-311.

Zhu, L., Rorai, C., Dhrubaditya, M. \& Brandt, L. 2014 A microfluidic device to sort capsules by deformability: a numerical study. Soft Matt. 10 (39), 7705-7711. 